\title{
A Mathematical Overview of the Monogamous Marriage in a Multiregion Framework: Modelling and Control
}

\author{
Mustapha Lhous $\mathbb{D}^{1},{ }^{1}$ Omar Zakary $\mathbb{D D}^{2},{ }^{2}$ and Mostafa Rachik ${ }^{2}{ }^{2}$ \\ ${ }^{1}$ Department of Mathematics and Computer Science, Faculty of Sciences Ain Chock, University Hassan II Casablanca, \\ Casablanca, Morocco \\ ${ }^{2}$ Department of Mathematics and Computer Science, Faculty of Sciences Ben M'sik, University Hassan II Casablanca, \\ Casablanca, Morocco \\ Correspondence should be addressed to Mustapha Lhous; mlhous17@gmail.com
}

Received 21 April 2020; Revised 6 July 2020; Accepted 13 July 2020; Published 14 August 2020

Academic Editor: Genni Fragnelli

Copyright (C) 2020 Mustapha Lhous et al. This is an open access article distributed under the Creative Commons Attribution License, which permits unrestricted use, distribution, and reproduction in any medium, provided the original work is properly cited.

\begin{abstract}
The main objective of this paper is to develop a new mathematical model to study, analyze, and control the family status in several regions and to discuss the impact of the connectivity of regions and the mobility of residents on the marital status of the family, by adopting a multiregion discrete-time model. The modelling and the control process of the system that describes the case of monogamous marriages in a multiregion framework are considered. Two combined control strategies are proposed, which allow reducing the virgin and divorced individuals and increasing the number of married individuals in a specific region. The first control is considered as the impact of public awareness campaigns to educate virgin men and women about the benefits of marriage for the individual and the society; the second control characterizes the legal procedures, administrative complications, and the heavy financial and social consequences of divorces. The optimal control theory is applied to characterize such optimal strategies and determined numerically using a progressive-regressive discrete scheme to discuss the obtained results.
\end{abstract}

\section{Introduction}

Mathematical models are useful tools for understanding the functioning of natural systems and for predicting their evolution. Among these models are those that study the dynamics of populations and ecosystems. Many researchers have studied models of population dynamics: prey and predator dynamics [1-4], epidemic dynamics in a population [5-8], molecular systems [9-11], and so forth.

Civil status is the situation of the person in the family and/or society. A person's marital status is positioned in one of four categories: virgin, married, divorced, or widowed (VMDW). Several authors have discussed the social functioning, marital status, family stability, and social control of health behavior [12-18]. In [19], the authors have given a discrete mathematical model that describes the marital status of the monogamous marriage case and they also studied the optimal control that reduces the number of unmarried and divorced people and increases the number of married people. In this paper, we generalize the results established in [19] to the case of a multiregion system. We studied the effect of the population travel to different regions for the marriage, divorce, and widow.

Many studies on civil status have given statistics on the family situation of different regions (cities, rural regions, industrial regions, tourist regions, etc.), according to the age group of the population. For example in Morocco, the High Commissioner for Planning (HCP, public institution) [14] gives different statistics according to the age groups of the population; there are $76.2 \%$ of single people for the age group of 20 to 24 and $54 \%$ for the group of 25 to 29 years old. The percentage of divorce is $30 \%$ for couples with less than 5 years of marriage and 3\% for married couples who have exceeded 20 years of marriages. $41.4 \%$ of married men and 
$41.8 \%$ of married women are identified by the HCP in the urban areas. The percentage of divorce is $0.8 \%$ for men and $3.1 \%$ for women. The widowed men are $0.6 \%$ of the population and women are $7.3 \%$ in urban areas. For the rural area, there are $40 \%$ of married men and $42.5 \%$ of married women; the percentage of divorce is $0.4 \%$ for married men and $1.4 \%$ for married women. Widowed men are represented by $0.6 \%$ of the population and women by $6.8 \%$ in rural areas.

In recent years, many attempts have been made to develop control strategies for different systems [5, 20-27]. There are a number of different methods for calculating the optimal control for a specific mathematical model. For example, Pontryagin et al.'s maximum principle [28] allows the characterization of the optimal control for an ordinary equation model system with a given constraint.

In [29], the authors have described a new modelling approach based on multiregion discrete-time SIR models aiming to describe the spatial-temporal evolution of epidemics that emerge in different geographical regions and to show the influence of one region on another region via infection travel.

In this paper and inspired by [29], we investigate an approach that determines an optimal control relative to a discrete VMDW model in a multiregion framework, which defines the evolution of the marital status of the marriage in a population, enabling decision-makers to develop very useful control strategies to reduce the virgin individuals and to increase the number of married individuals in a specific region.

The first control can be considered as public awareness campaigns showing to individuals the benefits of marriage on the psychic and social stability on persons and the society, or cultural entertained events to give people the chance to meet and to know and to allow themselves to get married. The second control is determined for the persons who have initiated the divorce proceedings; this control is considered as a long and costly legal procedure.
The optimal control problem was the subject of an optimization criterion represented by the minimization of an objective function. The optimality system is solved based on an iterative discrete schema that converges following an appropriate test similar to the one related to the ForwardBackward Sweep Method (FBSM).

The paper is organized as follows: in Section 2, the model VMDW is described for a multiregion discrete model. In Section 3, we give some results concerning the existence of optimal control and we use Pontryagin's maximum principle to investigate the analysis of control strategies and to determine the necessary condition for optimal control. Numerical simulations are given in Section 4. Finally, we conclude the paper in Section 5.

\section{Mathematical Modelling}

We consider a discrete-time model VMDW of the marital status of the family dynamic within a domain of interest $\Omega$ which represents a country, a city, a town, or a small domain. We assume that there are $p$ geographical regions (domains) of the domain studied $\Omega$. Let $\Omega=\cup_{j=1}^{p} \Omega_{j}$, and let $N_{i}\left(\Omega_{k}\right)$ be the population of domain $\Omega_{k}$ at time $i$; that is, the number of individuals who are residents in $\Omega_{k}$.

This model classifies the marital status of the family dynamics of a population into eight compartments in each region $\Omega_{k}$ : virgin men $V^{M}\left(\Omega_{k}\right)$, virgin women $V^{W}\left(\Omega_{k}\right)$, married men $M^{M}\left(\Omega_{k}\right)$, married women $M^{W}\left(\Omega_{k}\right)$, divorced men $D^{M}\left(\Omega_{k}\right)$, divorced women $D^{W}\left(\Omega_{k}\right)$, widowed men $W^{M}\left(\Omega_{k}\right)$, and widowed women $W^{W}\left(\Omega_{k}\right)$.

The unit of time $i$ can correspond to days, months, or years; it depends on the frequency of the survey and demographic studies as needed. However demographic statistics are generally done annually so the units $i, i+1, \ldots$ can be considered as years. The following system describes a discrete model of the marital status of the monogamous marriage case of a region $\Omega_{k}$ :

$$
\begin{aligned}
V_{i+1}^{M}\left(\Omega_{k}\right)= & \Lambda_{k 1}+V_{i}^{M}\left(\Omega_{k}\right)-\sum_{j=1}^{p} \frac{\alpha_{k j} V_{i}^{W}\left(\Omega_{j}\right)+\gamma_{k j} D_{i}^{W}\left(\Omega_{j}\right)+\delta_{k j} W_{i}^{W}\left(\Omega_{j}\right)}{N_{i}\left(\Omega_{k}\right)+N_{i}\left(\Omega_{j}\right)} V_{i}^{M}\left(\Omega_{k}\right) \\
& -d_{k} V_{i}^{M}\left(\Omega_{k}\right), \\
V_{i+1}^{W}\left(\Omega_{k}\right)= & \Lambda_{k 2}+V_{i}^{W}\left(\Omega_{k}\right)-\sum_{j=1}^{p} \frac{\alpha_{j k} V_{i}^{M}\left(\Omega_{j}\right)+\beta_{j k} D_{i}^{M}\left(\Omega_{j}\right)+\eta_{j k} W_{i}^{M}\left(\Omega_{j}\right)}{N_{i}\left(\Omega_{j}\right)+N_{i}\left(\Omega_{k}\right)} V_{i}^{W}\left(\Omega_{k}\right) \\
& -d_{k} V_{i}^{W}\left(\Omega_{k}\right), \\
D_{i+1}^{M}\left(\Omega_{k}\right)= & D_{i}^{M}\left(\Omega_{k}\right)-\sum_{j=1}^{p} \frac{\beta_{k j} V_{i}^{W}\left(\Omega_{j}\right)+\mu_{k j} D_{i}^{W}\left(\Omega_{j}\right)+\theta_{k j} W_{i}^{W}\left(\Omega_{j}\right)}{N_{i}\left(\Omega_{k}\right)+N_{i}\left(\Omega_{j}\right)} D_{i}^{M}\left(\Omega_{k}\right) \\
& +\lambda_{k} M_{i}^{M}\left(\Omega_{k}\right)-d_{k} D_{i}^{M}\left(\Omega_{k}\right),
\end{aligned}
$$




$$
\begin{aligned}
& D_{i+1}^{W}\left(\Omega_{k}\right)=D_{i}^{W}\left(\Omega_{k}\right)-\sum_{j=1}^{p} \frac{\gamma_{j k} V_{i}^{M}\left(\Omega_{j}\right)+\mu_{j k} D_{i}^{M}\left(\Omega_{j}\right)+v_{j k} W_{i}^{M}\left(\Omega_{j}\right)}{N_{i}\left(\Omega_{j}\right)+N_{i}\left(\Omega_{k}\right)} D_{i}^{W}\left(\Omega_{k}\right) \\
& +\sum_{j=1}^{p} \lambda_{j}^{k} M_{i}^{W}\left(\Omega_{j}\right)-d_{k} D_{i}^{W}\left(\Omega_{k}\right) \\
& W_{i+1}^{M}\left(\Omega_{k}\right)=W_{i}^{M}\left(\Omega_{k}\right)-\sum_{j=1}^{p} \frac{\eta_{k j} V_{i}^{W}\left(\Omega_{j}\right)+\nu_{k j} D_{i}^{W}\left(\Omega_{j}\right)+\sigma_{k j} W_{i}^{W}\left(\Omega_{j}\right)}{N_{i}\left(\Omega_{k}\right)+N_{i}\left(\Omega_{j}\right)} W_{i}^{M}\left(\Omega_{k}\right) \\
& +\omega_{k} M_{i}^{W}\left(\Omega_{k}\right)-d_{k} W_{i}^{M}\left(\Omega_{k}\right) \text {, } \\
& W_{i+1}^{W}\left(\Omega_{k}\right)=W_{i}^{W}\left(\Omega_{k}\right)-\sum_{j=1}^{p} \frac{\delta_{j k} V_{i}^{M}\left(\Omega_{j}\right)+\theta_{j k} D_{i}^{M}\left(\Omega_{j}\right)+\sigma_{j k} W_{i}^{M}\left(\Omega_{j}\right)}{N_{i}\left(\Omega_{j}\right)+N_{i}\left(\Omega_{k}\right)} W_{i}^{W}\left(\Omega_{k}\right) \\
& +\sum_{j=1}^{p} \rho_{j}^{k} M_{i}^{M}\left(\Omega_{j}\right)-d_{k} W_{i}^{W}\left(\Omega_{k}\right) \\
& M_{i+1}^{M}\left(\Omega_{k}\right)=M_{i}^{M}\left(\Omega_{k}\right)+\sum_{j=1}^{p}\left[\begin{array}{c}
\frac{\alpha_{k j}^{k} V_{i}^{W}\left(\Omega_{j}\right)+\gamma_{k j}^{k} D_{i}^{W}\left(\Omega_{j}\right)+\delta_{k j}^{k} W_{i}^{W}\left(\Omega_{j}\right)}{N_{i}\left(\Omega_{k}\right)+N_{i}\left(\Omega_{j}\right)} V_{i}^{M}\left(\Omega_{k}\right) \\
+\frac{\alpha_{j k}^{k} V_{i}^{M}\left(\Omega_{j}\right)+\beta_{j k}^{k} D_{i}^{M}\left(\Omega_{j}\right)+\eta_{j k}^{k} W_{i}^{M}\left(\Omega_{j}\right)}{N_{i}\left(\Omega_{j}\right)+N_{i}\left(\Omega_{j}\right)} V_{i}^{W}\left(\Omega_{k}\right) \\
+\frac{\beta_{k j}^{k} V_{i}^{W}\left(\Omega_{j}\right)+\mu_{k j}^{k} D_{i}^{W}\left(\Omega_{j}\right)+\theta_{k j}^{k} W_{i}^{W}\left(\Omega_{j}\right)}{N_{i}\left(\Omega_{j}\right)+\mu_{j k}^{k} D_{i}^{M}\left(\Omega_{j}\right)+v_{j k}^{k} W_{i}^{M}\left(\Omega_{j}\right)} D_{i}^{W}\left(\Omega_{k}\right) \\
N_{i}\left(\Omega_{j}\right)+N_{i}\left(\Omega_{k}\right) \\
+\frac{\eta_{k j}^{k} V_{i}^{W}\left(\Omega_{j}\right)+v_{k j}^{k} D_{i}^{W}\left(\Omega_{j}\right)+\sigma_{k j}^{k} W_{i}^{W}\left(\Omega_{j}\right)}{N_{i}\left(\Omega_{k}\right)+N_{i}\left(\Omega_{j}\right)} W_{i}^{M}\left(\Omega_{k}\right) \\
+\frac{\delta_{j k}^{k} V_{i}^{M}\left(\Omega_{j}\right)+\theta_{j k}^{k} D_{i}^{M}\left(\Omega_{j}\right)+\sigma_{j k}^{k} W_{i}^{M}\left(\Omega_{j}\right)}{N_{i}\left(\Omega_{j}\right)+N_{i}\left(\Omega_{k}\right)} W_{i}^{W}\left(\Omega_{k}\right)
\end{array}\right]-\left(\lambda_{k}+\rho_{k}\right) M_{i}^{M}\left(\Omega_{k}\right), \\
& M_{i+1}^{W}\left(\Omega_{k}\right)=M_{i}^{W}\left(\Omega_{k}\right)+\sum_{j=1}^{p}\left[\begin{array}{c}
\frac{\alpha_{k j}^{k} V_{i}^{W}\left(\Omega_{j}\right)+\gamma_{k j}^{k} D_{i}^{W}\left(\Omega_{j}\right)+\delta_{k j}^{k} W_{i}^{W}\left(\Omega_{j}\right)}{N_{i}\left(\Omega_{k}\right)+N_{i}\left(\Omega_{j}\right)} V_{i}^{M}\left(\Omega_{k}\right) \\
+\frac{\alpha_{j k}^{k} V_{i}^{M}\left(\Omega_{j}\right)+\beta_{j k}^{k} D_{i}^{M}\left(\Omega_{j}\right)+\eta_{j k}^{k} W_{i}^{M}\left(\Omega_{j}\right)}{N_{i}\left(\Omega_{j}\right)+N_{i}\left(\Omega_{k}\right)} V_{i}^{W}\left(\Omega_{k}\right) \\
+\frac{\beta_{j k}^{k} V_{i}^{W}\left(\Omega_{j}\right)+\mu_{k j}^{k} D_{i}^{W}\left(\Omega_{j}\right)+\theta_{k j}^{k} W_{i}^{W}\left(\Omega_{j}\right)}{N_{i}\left(\Omega_{k}\right)+N_{i}\left(\Omega_{j}\right)} D_{i}^{M}\left(\Omega_{k}\right) \\
N_{i}\left(\Omega_{j}\right)+N_{i}\left(\Omega_{k}\right)+v_{j k}^{k} W_{i}^{M}\left(\Omega_{j}\right) \\
+\frac{\eta_{k j}^{k} V_{i}^{W}\left(\Omega_{j}\right)+v_{k j}^{k} D_{i}^{W}\left(\Omega_{j}\right)+\sigma_{k j}^{k} W_{i}^{W}\left(\Omega_{j}\right)}{N_{i}\left(\Omega_{k}\right)+N_{i}\left(\Omega_{j}\right)} W_{i}^{M}\left(\Omega_{k}\right) \\
+\frac{\delta_{j k}^{k} V_{i}^{M}\left(\Omega_{j}\right)+\theta_{j k}^{k} D_{i}^{M}\left(\Omega_{j}\right)+\sigma_{j k}^{k} W_{i}^{M}\left(\Omega_{j}\right)}{N_{i}\left(\Omega_{j}\right)+N_{i}\left(\Omega_{k}\right)} W_{i}^{W}\left(\Omega_{k}\right)
\end{array}\right]-\left(\lambda_{k}+\omega_{k}\right) M_{i}^{W}\left(\Omega_{k}\right),
\end{aligned}
$$


TABLE 1: The description of parameters used for the definition of discrete-time systems (1)-(8).

\begin{tabular}{|c|c|}
\hline Parameter & Description \\
\hline$\Lambda_{k 1}$ & Natality of virgin men in region $\Omega_{k}$ \\
\hline$\Lambda_{k 2}$ & Natality of virgin women in region $\Omega_{k}$ \\
\hline$d_{k}$ & Natural mortality rate in region $\Omega_{k}$ \\
\hline$\alpha_{k j}$ & Marriage rate of virgin men of region $\Omega_{k}$ to virgin women of region $\Omega_{j}$ \\
\hline$\beta_{k j}$ & Marriage rate of divorced men of region $\Omega_{k}$ to virgin women of region $\Omega_{j}$ \\
\hline$\gamma_{k j}$ & Marriage rate of virgin men of region $\Omega_{k}$ to divorced women of region $\Omega_{j}$ \\
\hline$\delta_{k j}$ & Marriage rate of virgin men of region $\Omega_{k}$ to widowed women of region $\Omega_{j}$ \\
\hline$\eta_{k j}$ & Marriage rate of widowed men of region $\Omega_{k}$ to virgin women of region $\Omega_{j}$ \\
\hline$\mu_{k j}$ & Marriage rate of divorced men of region $\Omega_{k}$ to divorced women of region $\Omega_{j}$ \\
\hline$\theta_{k j}$ & Marriage rate of divorced men of region $\Omega_{k}$ to widowed women of region $\Omega_{j}$ \\
\hline$v_{k j}$ & Marriage rate of widowed men of region $\Omega_{k}$ to divorced women of region $\Omega_{j}$ \\
\hline$\sigma_{k j}$ & Marriage rate of widowed men of region $\Omega_{k}$ to widowed women of region $\Omega_{j}$ \\
\hline$\lambda_{k}$ & Divorce rate of married men of region $\Omega_{k}$ \\
\hline$\lambda_{j}^{k}$ & Divorce rate of women of region $\Omega_{k}$ which return to the region $\Omega_{j}$ \\
\hline$\rho_{k}$ & Widow rate of married women of region $\Omega_{k}$ \\
\hline$\omega_{k}$ & Widow rate of married men of region $\Omega_{k}$ \\
\hline
\end{tabular}

where $V_{0}^{M}\left(\Omega_{k}\right), V_{0}^{W}\left(\Omega_{k}\right), M_{0}^{M}\left(\Omega_{k}\right), M_{0}^{W}\left(\Omega_{k}\right), D_{0}^{M}\left(\Omega_{k}\right)$, $D_{0}^{W}\left(\Omega_{k}\right), W_{0}^{M}\left(\Omega_{k}\right)$, and $W_{0}^{W}\left(\Omega_{k}\right)$ are the given initial state in the region $\Omega_{k}$. In equations (1)-(8), all parameters are nonnegative and defined in Table 1.

For equation (1) of the model, virgin men of a region $\Omega_{k}$ can contact a virgin, divorced, or widowed woman of another region $\Omega_{j}$ with $\alpha_{k j}, \gamma_{k j}$, and $\delta_{k j}$ rates, respectively, and this contact can result in a marriage. The married couple can stay in the region $\Omega_{k}$ or settle in the conjoin region $\Omega_{j}$. Thus, the number of virgin men decreases and the number of virgins at the instant $i$ is substituted for the number $\alpha_{k j} V_{i}^{M}\left(\Omega_{k}\right) V_{i}^{W}\left(\Omega_{j}\right) / N_{i}\left(\Omega_{k}\right)+N_{i}\left(\Omega_{j}\right)+\gamma_{k j} V_{i}^{M}\left(\Omega_{k}\right) D_{i}^{W}$ $\left(\Omega_{j}\right) / N_{i}\left(\Omega_{k}\right)+N_{i}\left(\Omega_{j}\right)+\delta_{k j} V_{i}^{M}\left(\Omega_{k}\right) W_{i}^{W}\left(\Omega_{j}\right) / N_{i}\left(\Omega_{k}\right)+$ $N_{i}\left(\Omega_{j}\right)$ and a portion of this number $\alpha_{k j}^{k} V_{i}^{M}\left(\Omega_{k}\right)$ $V_{i}^{W}\left(\Omega_{j}\right) / N_{i}\left(\Omega_{k}\right)+N_{i}\left(\Omega_{j}\right)+\gamma_{k j}^{k} V_{i}^{M}\left(\Omega_{k}\right) D_{i}^{W}\left(\Omega_{j}\right) / N_{i}\left(\Omega_{k}\right)+$ $N_{i}\left(\Omega_{j}\right)+\delta_{k j}^{k} V_{i}^{M}\left(\Omega_{k}\right) W_{i}^{W}\left(\Omega_{j}\right) / N_{i}\left(\Omega_{k}\right)+N_{i}\left(\Omega_{j}\right)$ that represents the part of married couples who decides to settle in $\Omega_{k}$ is added at the time $i+1$ to the number of men marrying in region $\Omega_{k}$ and the other portion $\alpha_{k j}^{j} V_{i}^{M}$ $\left(\Omega_{k}\right) V_{i}^{W}\left(\Omega_{j}\right) / N_{i}\left(\Omega_{k}\right)+N_{i}\left(\Omega_{j}\right)+\gamma_{k j}^{j} V_{i}^{M}\left(\Omega_{k}\right) D_{i}^{W}\left(\Omega_{j}\right) / N_{i}$ $\left(\Omega_{k}\right)+N_{i}\left(\Omega_{j}\right)+\delta_{k j}^{j} V_{i}^{M}\left(\Omega_{k}\right) W_{i}^{W}\left(\Omega_{j}\right) / N_{i}\left(\Omega_{k}\right)+N_{i}\left(\Omega_{j}\right)$ is added to the number of men marrying in region $\Omega_{j}$. Then, we have $\alpha_{k j}=\alpha_{k j}^{k}+\alpha_{k j}^{j}, \gamma_{k j}=\gamma_{k j}^{k}+\gamma_{k j}^{j}$ and $\delta_{k j}=\delta_{k j}^{k}+\delta_{k j}^{j}$.

Similarly in equation (2), the number of virgin women in region $\Omega_{k}$ decreases at the instant $i+1$ by substituting the number of virgin women at the instant $i$, the number $\alpha_{j k} V_{i}^{M}\left(\Omega_{j}\right) V_{i}^{W}\left(\Omega_{k}\right) / N_{i}\left(\Omega_{j}\right)+N_{i}\left(\Omega_{k}\right)+\beta_{j k} D_{i}^{M}\left(\Omega_{j}\right) V_{i}^{W}$ $\left(\Omega_{k}\right) / N_{i}\left(\Omega_{j}\right)+N_{i}\left(\Omega_{k}\right)+\eta_{j k} W_{i}^{M}\left(\Omega_{j}\right) V_{i}^{W}\left(\Omega_{k}\right) / N_{i}\left(\Omega_{j}\right)+$ $N_{i}\left(\Omega_{k}\right)$ which represents the number of married women after contact with a virgin, divorced, or widowed men in region $\Omega_{j}$ with $\alpha_{j k}, \beta_{j k}$, and $\eta_{j k}$ levels, respectively. Also we have $\alpha_{j k}=\alpha_{j k}^{j}+\alpha_{j k}^{k}, \beta_{j k}=\beta_{j k}^{j}+\beta_{j k}^{k}$ and $\eta_{j k}=\eta_{j k}^{j}+\eta_{j k}^{k}$.

In equation (3), a divorced man in region $\Omega_{k}$ can contact a virgin, divorced, or widowed woman of region $\Omega_{j}$ with $\beta_{k j}$, $\mu_{k j}$, and $\theta_{k j}$ rates, respectively, so this contact can result in marriage. Then the number $\beta_{k j} D_{i}^{M}\left(\Omega_{k}\right) V_{i}^{W}\left(\Omega_{j}\right) / N_{i}\left(\Omega_{k}\right)+$ $N_{i}\left(\Omega_{j}\right)+\mu_{k j} D_{i}^{M}\left(\Omega_{k}\right) D_{i}^{W}\left(\Omega_{j}\right) / N_{i}\left(\Omega_{k}\right)+N_{i}\left(\Omega_{j}\right)+\theta_{k j} D_{i}^{M}$ $\left(\Omega_{k}\right) W_{i}^{W}\left(\Omega_{j}\right) / N_{i}\left(\Omega_{k}\right)+N_{i}\left(\Omega_{j}\right)$ is decreased by the number of divorced men at the time $i+1$ and added to the number of married men and we have $\mu_{k j}=\mu_{k j}^{k}+\mu_{k j}^{j}$ and $\theta_{k j}=\theta_{k j}^{k}+\theta_{k j}^{j}$.
In the model we propose here, it was considered that a divorced man remains in his region and the divorced woman returns to the region of her parents; this is the case for the majority of regions whether they are conservative or not. And so the number $\lambda_{k} M_{i}^{M}\left(\Omega_{k}\right)$ is added to the number of divorced men and the number $\lambda_{j}^{k} M_{i}^{W}\left(\Omega_{j}\right)$ of divorced women is added to the number of divorced women of the region $\Omega_{k}$ with $\lambda_{j}^{k}$ being the rate that a woman divorces a man from the region $\Omega_{k}$ and returns to the region $\Omega_{j}$. Consequently, the total number of $\sum_{j=1}^{p} \lambda_{j}^{k} M_{i}^{W}\left(\Omega_{j}\right)$ is added to the number of divorced women in the region $\Omega_{k}$ with the relation $\lambda_{k}=\sum_{j=1}^{p} \lambda_{k}^{j}$. The same principle applies to equations (5) and (6) with $\rho_{k}=\sum_{j=1}^{p} \rho_{k}^{j}$.

For equation (7), the number of married men in the region $\Omega_{k}$ increases at the instant $i+1$ by the number of virgin, divorced, and widowed men who are married by contacting virgin, divorced, or widowed women of the region $\Omega_{j}$ and decreases the natural mortality with a $\rho_{k}$ and divorce rate with a $\lambda_{k}$ rate. The same principle can be applied to equation (8).

In equations (7) and (8) from the model, the natural mortality of married men and women was considered. The mortality rate for married men is $\rho_{k}$ and the mortality rate for married women is $\omega_{k}$. These rates appear in equations (5) and (6) which correspond to widowed men and women, respectively, so the number of mortalities which is $\rho_{k} M_{i}^{M}\left(\Omega_{k}\right)$ married men and the number $\sum_{j=1}^{p} \rho_{j}^{k} M_{i}^{M}\left(\Omega_{j}\right)$ are added to the number of widowed women and similarly the number $\omega_{k} M_{i}^{W}\left(\Omega_{k}\right)$ is added to the number of widowed men.

\section{Methods and Results}

3.1. An Optimal Control Approach. An optimal control approach has been applied to models $(1)-(8)$ to reduce the virgin and divorced individuals and increase the number of married individuals along the control strategy period. For this, we introduce a control variable $\left(u_{i}, v_{i}\right)$ that characterizes the benefits of an awareness campaign to educate virgin men and women about the benefits of marriage for the individual and the society, especially the legal procedures, administrative complications, and the heavy financial and social consequences of divorces, respectively, in the abovementioned models (1)-(8). Then, the model is given by the following equations: 


$$
\begin{aligned}
& V_{i+1}^{M}\left(\Omega_{k}\right)=\Lambda_{k 1}+V_{i}^{M}\left(\Omega_{k}\right)-\sum_{j=1}^{p} \frac{\alpha_{k j} V_{i}^{W}\left(\Omega_{j}\right)+\gamma_{k j} D_{i}^{W}\left(\Omega_{j}\right)+\delta_{k j} W_{i}^{W}\left(\Omega_{j}\right)}{N_{i}\left(\Omega_{k}\right)+N_{i}\left(\Omega_{j}\right)} V_{i}^{M}\left(\Omega_{k}\right) \\
& -d_{k} V_{i}^{M}\left(\Omega_{k}\right)-u_{i} V_{i}^{M}\left(\Omega_{k}\right), \\
& V_{i+1}^{W}\left(\Omega_{k}\right)=\Lambda_{k 2}+V_{i}^{W}\left(\Omega_{k}\right)-\sum_{j=1}^{p} \frac{\alpha_{j k} V_{i}^{M}\left(\Omega_{j}\right)+\beta_{j k} D_{i}^{M}\left(\Omega_{j}\right)+\eta_{j k} W_{i}^{M}\left(\Omega_{j}\right)}{N_{i}\left(\Omega_{j}\right)+N_{i}\left(\Omega_{k}\right)} V_{i}^{W}\left(\Omega_{k}\right) \\
& -d_{k} V_{i}^{W}\left(\Omega_{k}\right)-u_{i} V_{i}^{W}\left(\Omega_{k}\right) \\
& D_{i+1}^{M}\left(\Omega_{k}\right)=D_{i}^{M}\left(\Omega_{k}\right)-\sum_{j=1}^{p} \frac{\beta_{k j} V_{i}^{W}\left(\Omega_{j}\right)+\mu_{k j} D_{i}^{W}\left(\Omega_{j}\right)+\theta_{k j} W_{i}^{W}\left(\Omega_{j}\right)}{N_{i}\left(\Omega_{k}\right)+N_{i}\left(\Omega_{j}\right)} D_{i}^{M}\left(\Omega_{k}\right) \\
& +\lambda_{k} M_{i}^{M}\left(\Omega_{k}\right)-d_{k} D_{i}^{M}\left(\Omega_{k}\right)-v_{i} D_{i}^{M}\left(\Omega_{k}\right), \\
& D_{i+1}^{W}\left(\Omega_{k}\right)=D_{i}^{W}\left(\Omega_{k}\right)-\sum_{j=1}^{p} \frac{\gamma_{j k} V_{i}^{M}\left(\Omega_{j}\right)+\mu_{j k} D_{i}^{M}\left(\Omega_{j}\right)+v_{j k} W_{i}^{M}\left(\Omega_{j}\right)}{N_{i}\left(\Omega_{j}\right)+N_{i}\left(\Omega_{k}\right)} D_{i}^{W}\left(\Omega_{k}\right) \\
& +\sum_{j=1}^{p} \lambda_{j}^{k} M_{i}^{W}\left(\Omega_{j}\right)-d_{k} D_{i}^{W}\left(\Omega_{k}\right)-v_{i} D_{i}^{W}\left(\Omega_{k}\right) \\
& W_{i+1}^{M}\left(\Omega_{k}\right)=W_{i}^{M}\left(\Omega_{k}\right)-\sum_{j=1}^{p} \frac{\eta_{k j} V_{i}^{W}\left(\Omega_{j}\right)+v_{k j} D_{i}^{W}\left(\Omega_{j}\right)+\sigma_{k j} W_{i}^{W}\left(\Omega_{j}\right)}{N_{i}\left(\Omega_{k}\right)+N_{i}\left(\Omega_{j}\right)} W_{i}^{M}\left(\Omega_{k}\right) \\
& +\omega_{k} M_{i}^{W}\left(\Omega_{k}\right)-d_{k} W_{i}^{M}\left(\Omega_{k}\right), \\
& W_{i+1}^{W}\left(\Omega_{k}\right)=W_{i}^{W}\left(\Omega_{k}\right)-\sum_{j=1}^{p} \frac{\delta_{j k} V_{i}^{M}\left(\Omega_{j}\right)+\theta_{j k} D_{i}^{M}\left(\Omega_{j}\right)+\sigma_{j k} W_{i}^{M}\left(\Omega_{j}\right)}{N_{i}\left(\Omega_{j}\right)+N_{i}\left(\Omega_{k}\right)} W_{i}^{W}\left(\Omega_{k}\right) \\
& +\sum_{j=1}^{p} \rho_{j}^{k} M_{i}^{M}\left(\Omega_{j}\right)-d_{k} W_{i}^{W}\left(\Omega_{k}\right)
\end{aligned}
$$

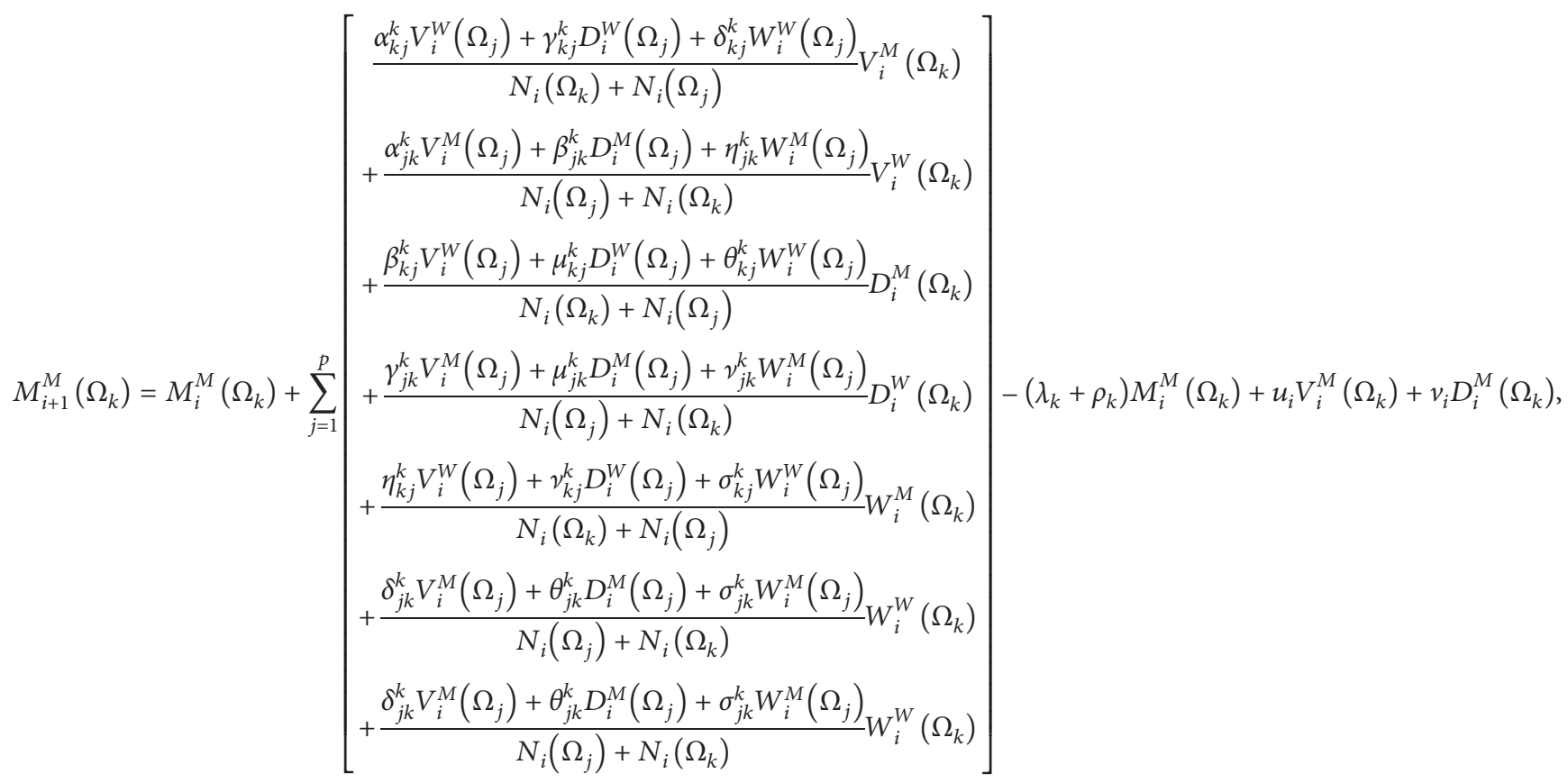




$$
M_{i+1}^{W}\left(\Omega_{k}\right)=M_{i}^{W}\left(\Omega_{k}\right)+\sum_{j=1}^{p}\left[\begin{array}{c}
\frac{\alpha_{k j}^{k} V_{i}^{W}\left(\Omega_{j}\right)+\gamma_{k j}^{k} D_{i}^{W}\left(\Omega_{j}\right)+\delta_{k j}^{k} W_{i}^{W}\left(\Omega_{j}\right)}{N_{i}\left(\Omega_{k}\right)+N_{i}\left(\Omega_{j}\right)} V_{i}^{M}\left(\Omega_{k}\right) \\
+\frac{\alpha_{j k}^{k} V_{i}^{M}\left(\Omega_{j}\right)+\beta_{j k}^{k} D_{i}^{M}\left(\Omega_{j}\right)+\eta_{j k}^{k} W_{i}^{M}\left(\Omega_{j}\right)}{N_{i}\left(\Omega_{j}\right)+N_{i}\left(\Omega_{k}\right)} V_{i}^{W}\left(\Omega_{k}\right) \\
+\frac{\beta_{k j}^{k} V_{i}^{W}\left(\Omega_{j}\right)+\mu_{k j}^{k} D_{i}^{W}\left(\Omega_{j}\right)+\theta_{k j}^{k} W_{i}^{W}\left(\Omega_{j}\right)}{N_{i}\left(\Omega_{k}\right)+N_{i}\left(\Omega_{j}\right)} D_{i}^{M}\left(\Omega_{k}\right) \\
+\frac{\gamma_{j k}^{k} V_{i}^{M}\left(\Omega_{j}\right)+\mu_{j k}^{k} D_{i}^{M}\left(\Omega_{j}\right)+v_{j k}^{k} W_{i}^{M}\left(\Omega_{j}\right)}{\left.N_{i}\right)} D_{i}^{W}\left(\Omega_{k}\right) \\
+\frac{\eta_{k j}^{k} V_{i}^{W}\left(\Omega_{j}\right)+v_{k j}^{k} D_{i}^{W}\left(\Omega_{j}\right)+\sigma_{k j}^{k} W_{i}^{W}\left(\Omega_{j}\right)}{N_{i}\left(\Omega_{k}\right)+N_{i}\left(\Omega_{j}\right)} W_{i}^{M}\left(\Omega_{k}\right) \\
N_{i}\left(\Omega_{j}\right)+N_{i}\left(\Omega_{k}\right) \\
+\frac{\delta_{j k}^{k} V_{i}^{M}\left(\Omega_{j}\right)+\theta_{j k}^{k} D_{i}^{M}\left(\Omega_{j}\right)+\sigma_{j k}^{k} W_{i}^{M}\left(\Omega_{j}\right)}{W_{i}^{W}\left(\Omega_{k}\right)}
\end{array}\right]-\left(\lambda_{k}+\omega_{k}\right) M_{i}^{W}\left(\Omega_{k}\right)+u_{i} V_{i}^{W}\left(\Omega_{k}\right)+v_{i} D_{i}^{W}\left(\Omega_{k}\right),
$$

3.2. Characterization of the Optimal Control. For an initial state $\left(V_{0}^{M}\left(\Omega_{k}\right), V_{0}^{W}\left(\Omega_{k}\right), M_{0}^{M}\left(\Omega_{k}\right), M_{0}^{W}\left(\Omega_{k}\right), \quad D_{0}^{M}\left(\Omega_{k}\right)\right.$, $\left.D_{0}^{W}\left(\Omega_{k}\right)\right)$, $\left(W_{0}^{M}\left(\Omega_{k}\right), W_{0}^{W}\left(\Omega_{k}\right)\right)$, we consider an optimization criterion defined by the following objective function:

$$
\begin{aligned}
J(u, v)= & \sum_{i=0}^{N}\left(A_{1} V_{i}^{W}\left(\Omega_{k}\right)+A_{2} D_{i}^{W}\left(\Omega_{k}\right)-A_{3} M_{i}^{W}\left(\Omega_{k}\right)\right) \\
& +\sum_{i=0}^{N-1}\left(\frac{\tau_{1}}{2}\left(u_{i}\right)^{2}+\frac{\tau_{2}}{2}\left(v_{i}\right)^{2}\right)
\end{aligned}
$$

subject to systems (9)-(16). Here, $A_{1}, A_{2}$, and $A_{3}$ are positive constants to keep a balance in the size of $V_{i}^{W}\left(\Omega_{k}\right), D_{i}^{W}\left(\Omega_{k}\right)$, and $M_{i}^{W}\left(\Omega_{k}\right)$, respectively. In the objective functional, $\tau_{1}$ and $\tau_{2}$ are the positive weight parameters which are associated with the controls $u_{i}$ and $v_{i}$.
In other words, we seek the optimal controls $\left(u^{*}, v^{*}\right)$ such that

$$
J\left(u^{*}, v^{*}\right)=\min \left\{J(u, v) \mid(u, v) \in \mathcal{U}_{a d}\right\},
$$

where $\mathcal{U}_{a d}$ is the set of admissible controls defined by

$$
\mathcal{U}_{a d}=\left\{(u, v) \mid u^{\min } \leq u_{i} \leq u^{\max }, v^{\min } \leq v_{i} \leq v^{\max }, i \in\{0, \ldots, N-1\}\right\},
$$

where $\left(u^{\min }, u^{\max }, v^{\min }, v^{\max }\right) \in[0,1]^{4}$.

The sufficient condition for existence of an optimal control $\left(u^{*}, v^{*}\right)$ for problem (18) follows from standard results of [21]. In order to find an optimal solution, first we find the Hamiltonian for the optimal control problem (18). In fact, the Hamiltonian $H$ of the optimal problem is given by 
Discrete Dynamics in Nature and Society

7

$$
\begin{aligned}
& H=A_{1} V_{i}^{W}\left(\Omega_{k}\right)+A_{2} D_{i}^{W}\left(\Omega_{k}\right)-A_{3} M_{i}^{W}\left(\Omega_{k}\right)+\frac{\tau_{1}}{2} u_{i}^{2}+\frac{\tau_{2}}{2} v_{i}^{2} \\
& +\zeta_{1, i+1}^{k}\left[\Lambda_{k 1}+V_{i}^{M}\left(\Omega_{k}\right)-\sum_{j=1}^{p} \frac{\alpha_{k j} V_{i}^{W}\left(\Omega_{j}\right)+\gamma_{k j} D_{i}^{W}\left(\Omega_{j}\right)+\delta_{k j} W_{i}^{W}\left(\Omega_{j}\right)}{N_{i}\left(\Omega_{k}\right)+N_{i}\left(\Omega_{j}\right)} V_{i}^{M}\left(\Omega_{k}\right)-d_{k} V_{i}^{M}\left(\Omega_{k}\right)-u_{i} V_{i}^{M}\left(\Omega_{k}\right)\right] \\
& +\zeta_{2, i+1}^{k}\left[\Lambda_{k 2}+V_{i}^{W}\left(\Omega_{k}\right)-\sum_{j=1}^{p} \frac{\alpha_{j k} V_{i}^{M}\left(\Omega_{j}\right)+\beta_{j k} D_{i}^{M}\left(\Omega_{j}\right)+\eta_{j k} W_{i}^{M}\left(\Omega_{j}\right)}{N_{i}\left(\Omega_{j}\right)+N_{i}\left(\Omega_{k}\right)} V_{i}^{W}\left(\Omega_{k}\right)-d_{k} V_{i}^{W}\left(\Omega_{k}\right)-u_{i} V_{i}^{W}\left(\Omega_{k}\right)\right] \\
& +\zeta_{3, i+1}^{k}\left[D_{i}^{M}\left(\Omega_{k}\right)-\sum_{j=1}^{p} \frac{\beta_{k j} V_{i}^{W}\left(\Omega_{j}\right)+\mu_{k j} D_{i}^{W}\left(\Omega_{j}\right)+\theta_{k j} W_{i}^{W}\left(\Omega_{j}\right)}{N_{i}\left(\Omega_{k}\right)+N_{i}\left(\Omega_{j}\right)} D_{i}^{M}\left(\Omega_{k}\right)+\lambda_{k} M_{i}^{M}\left(\Omega_{k}\right)-d_{k} D_{i}^{M}\left(\Omega_{k}\right)-v_{i} D_{i}^{M}\left(\Omega_{k}\right)\right] \\
& +\zeta_{4, i+1}^{k}\left[D_{i}^{W}\left(\Omega_{k}\right)-\sum_{j=1}^{p} \frac{\gamma_{j k} V_{i}^{M}\left(\Omega_{j}\right)+\mu_{j k} D_{i}^{M}\left(\Omega_{j}\right)+v_{j k} W_{i}^{M}\left(\Omega_{j}\right)}{N_{i}\left(\Omega_{j}\right)+N_{i}\left(\Omega_{k}\right)} D_{i}^{W}\left(\Omega_{k}\right)+\sum_{j=1}^{p} \lambda_{j}^{k} M_{i}^{W}\left(\Omega_{j}\right)-d_{k} D_{i}^{W}\left(\Omega_{k}\right)-v_{i} D_{i}^{W}\left(\Omega_{k}\right)\right] \\
& +\zeta_{5, i+1}^{k}\left[W_{i}^{M}\left(\Omega_{k}\right)-\sum_{j=1}^{p} \frac{\eta_{k j} V_{i}^{W}\left(\Omega_{j}\right)+v_{k j} D_{i}^{W}\left(\Omega_{j}\right)+\sigma_{k j} W_{i}^{W}\left(\Omega_{j}\right)}{N_{i}\left(\Omega_{k}\right)+N_{i}\left(\Omega_{j}\right)} W_{i}^{M}\left(\Omega_{k}\right)+\omega_{k} M_{i}^{W}\left(\Omega_{k}\right)-d_{k} W_{i}^{M}\left(\Omega_{k}\right)\right] \\
& +\zeta_{6, i+1}^{k}\left[W_{i}^{W}\left(\Omega_{k}\right)-\sum_{j=1}^{p} \frac{\delta_{j k} V_{i}^{M}\left(\Omega_{j}\right)+\theta_{j k} D_{i}^{M}\left(\Omega_{j}\right)+\sigma_{j k} W_{i}^{M}\left(\Omega_{j}\right)}{N_{i}\left(\Omega_{j}\right)+N_{i}\left(\Omega_{k}\right)} W_{i}^{W}\left(\Omega_{k}\right)+\sum_{j=1}^{p} \rho_{j}^{k} M_{i}^{M}\left(\Omega_{j}\right)-d_{k} W_{i}^{W}\left(\Omega_{k}\right)\right] \\
& +\zeta_{7, i+1}^{k}\left[\begin{array}{c}
\frac{\alpha_{k j}^{k} V_{i}^{W}\left(\Omega_{j}\right)+\gamma_{k j}^{k} D_{i}^{W}\left(\Omega_{j}\right)+\delta_{k j}^{k} W_{i}^{W}\left(\Omega_{j}\right)}{N_{i}\left(\Omega_{k}\right)+N_{i}\left(\Omega_{j}\right)} V_{i}^{M}\left(\Omega_{k}\right) \\
+\frac{\alpha_{j k}^{k} V_{i}^{M}\left(\Omega_{j}\right)+\beta_{j k}^{k} D_{i}^{M}\left(\Omega_{j}\right)+\eta_{j k}^{k} W_{i}^{M}\left(\Omega_{j}\right)}{N_{i}\left(\Omega_{j}\right)+N_{i}\left(\Omega_{k}\right)} V_{i}^{W}\left(\Omega_{k}\right) \\
M_{j=1}^{p}\left[\begin{array}{c}
+\frac{\beta_{k j}^{k} V_{i}^{W}\left(\Omega_{j}\right)+\mu_{k j}^{k} D_{i}^{W}\left(\Omega_{j}\right)+\theta_{k j}^{k} W_{i}^{W}\left(\Omega_{j}\right)}{N_{i}\left(\Omega_{k}\right)+N_{i}\left(\Omega_{j}\right)} D_{i}^{M}\left(\Omega_{k}\right) \\
+\frac{\gamma_{j k}^{k} V_{i}^{M}\left(\Omega_{j}\right)+\mu_{j k}^{k} D_{i}^{M}\left(\Omega_{j}\right)+\gamma_{j k}^{k} W_{i}^{M}\left(\Omega_{j}\right)}{N_{i}\left(\Omega_{j}\right)+N_{i}\left(\Omega_{k}\right)} D_{i}^{W}\left(\Omega_{k}\right) \\
+\frac{\eta_{k j}^{k} V_{i}^{W}\left(\Omega_{j}\right)+v_{k j}^{k} D_{i}^{W}\left(\Omega_{j}\right)+\sigma_{k j}^{k} W_{i}^{W}\left(\Omega_{j}\right)}{N_{i}\left(\Omega_{k}\right)+N_{i}\left(\Omega_{j}\right)} W_{i}^{M}\left(\Omega_{k}\right) \\
+\frac{\delta_{j k}^{k} V_{i}^{M}\left(\Omega_{j}\right)+\theta_{j k}^{k} D_{i}^{M}\left(\Omega_{j}\right)+\sigma_{j k}^{k} W_{i}^{M}\left(\Omega_{j}\right)}{N_{i}\left(\Omega_{j}\right)+N_{i}\left(\Omega_{k}\right)} W_{i}^{W}\left(\Omega_{k}\right)
\end{array}\right]-\left(\lambda_{k}+\rho_{k}\right) M_{i}^{M}\left(\Omega_{k}\right)+u_{i} V_{i}^{M}\left(\Omega_{k}\right)+v_{i} D_{i}^{M}\left(\Omega_{k}\right)
\end{array}\right] \\
& +\zeta_{8, i+1}^{k}\left[\begin{array}{c}
\frac{\alpha_{k j}^{k} V_{i}^{W}\left(\Omega_{j}\right)+\gamma_{k j}^{k} D_{i}^{W}\left(\Omega_{j}\right)+\delta_{k j}^{k} W_{i}^{W}\left(\Omega_{j}\right)}{N_{i}\left(\Omega_{k}\right)+N_{i}\left(\Omega_{j}\right)} V_{i}^{M}\left(\Omega_{k}\right) \\
+\frac{\alpha_{j k}^{k} V_{i}^{M}\left(\Omega_{j}\right)+\beta_{j k}^{k} D_{i}^{M}\left(\Omega_{j}\right)+\eta_{j k}^{k} W_{i}^{M}\left(\Omega_{j}\right)}{N_{i}\left(\Omega_{j}\right)+N_{i}\left(\Omega_{k}\right)} V_{i}^{W}\left(\Omega_{k}\right) \\
\sum_{j=1}^{p}\left[\begin{array}{c}
+\frac{\beta_{k j}^{k} V_{i}^{W}\left(\Omega_{j}\right)+\mu_{k j}^{k} D_{i}^{W}\left(\Omega_{j}\right)+\theta_{k j}^{k} W_{i}^{W}\left(\Omega_{j}\right)}{N_{i}\left(\Omega_{k}\right)+N_{i}\left(\Omega_{j}\right)} D_{i}^{M}\left(\Omega_{k}\right) \\
+\frac{\gamma_{j k}^{k} V_{i}^{M}\left(\Omega_{j}\right)+\mu_{j k}^{k} D_{i}^{M}\left(\Omega_{j}\right)+\gamma_{j k}^{k} W_{i}^{M}\left(\Omega_{j}\right)}{N_{i}\left(\Omega_{j}\right)+N_{i}\left(\Omega_{k}\right)} D_{i}^{W}\left(\Omega_{k}\right) \\
+\frac{\eta_{k j}^{k} V_{i}^{W}\left(\Omega_{j}\right)+\gamma_{k j}^{k} D_{i}^{W}\left(\Omega_{j}\right)+\sigma_{k j}^{k} W_{i}^{W}\left(\Omega_{j}\right)}{N_{i}\left(\Omega_{k}\right)+N_{i}\left(\Omega_{j}\right)} W_{i}^{M}\left(\Omega_{k}\right) \\
+\frac{\delta_{j k}^{k} V_{i}^{M}\left(\Omega_{j}\right)+\theta_{j k}^{k} D_{i}^{M}\left(\Omega_{j}\right)+\sigma_{j k}^{k} W_{i}^{M}\left(\Omega_{j}\right)}{N_{i}\left(\Omega_{j}\right)+N_{i}\left(\Omega_{k}\right)} W_{i}^{W}\left(\Omega_{k}\right)
\end{array}\right]-\left(\lambda_{k}+\omega_{k}\right) M_{i}^{W}\left(\Omega_{k}\right)+u_{i} V_{i}^{W}\left(\Omega_{k}\right)+v_{i} D_{i}^{W}\left(\Omega_{k}\right)
\end{array}\right],
\end{aligned}
$$


where $\zeta_{1}^{k}, \zeta_{2}^{k}, \ldots, \zeta_{8}^{k}$ are the adjoint functions to be determined suitably.

At the same time by using Pontryagin et al.'s maximum principle [28], we derive necessary conditions for our optimal control. We obtain the following theorem.
Theorem 1. Let $V^{M *}\left(\Omega_{k}\right), \quad V^{W *}\left(\Omega_{k}\right), \quad M^{M *}\left(\Omega_{k}\right)$, $M^{W *}\left(\Omega_{k}\right), \quad D^{M *}\left(\Omega_{k}\right), \quad D^{W *}\left(\Omega_{k}\right), \quad W^{M *}\left(\Omega_{k}\right), \quad$ and $W^{W *}\left(\Omega_{k}\right)$ be optimal state solutions with associated optimal control $\left(u^{*}, v^{*}\right)$ for the optimal control problem (18). Then, there exist adjoint variables $\zeta_{1}^{k}, \zeta_{2}^{k}, \ldots, \zeta_{8}^{k}$ that satisfy

$$
\begin{aligned}
& \Delta \zeta_{1, i}^{k}=-\zeta_{1, i+1}^{k}\left(1-\sum_{j=1}^{p} \frac{\alpha_{k j} V_{i}^{W}\left(\Omega_{j}\right)+\gamma_{k j} D_{i}^{W}\left(\Omega_{j}\right)+\delta_{k j} W_{i}^{W}\left(\Omega_{j}\right)}{N_{i}\left(\Omega_{k}\right)+N_{i}\left(\Omega_{j}\right)}-d_{k}-u_{i}\right) \\
& +\zeta_{2, i+1}^{k} \frac{\alpha_{k k} V_{i}^{W}\left(\Omega_{k}\right)}{2 N_{i}\left(\Omega_{k}\right)}+\zeta_{4, i+1}^{k} \frac{\gamma_{k k} D_{i}^{W}\left(\Omega_{k}\right)}{2 N_{i}\left(\Omega_{k}\right)}+\zeta_{6, i+1}^{k} \frac{\delta_{k k} W_{i}^{W}\left(\Omega_{k}\right)}{2 N_{i}\left(\Omega_{k}\right)} \\
& -\left(\zeta_{7, i+1}^{k}+\zeta_{8, i+1}^{k}\right)\left(\sum_{j=1}^{p} \frac{\alpha_{k j}^{k} V_{i}^{W}\left(\Omega_{j}\right)+\gamma_{k j}^{k} D_{i}^{W}\left(\Omega_{j}\right)+\delta_{k j}^{k} W_{i}^{W}\left(\Omega_{j}\right)}{N_{i}\left(\Omega_{k}\right)+N_{i}\left(\Omega_{j}\right)}+\frac{\alpha_{k k}^{k} V_{i}^{W}\left(\Omega_{k}\right)+\gamma_{k k}^{k} D_{i}^{W}\left(\Omega_{k}\right)+\delta_{k k}^{k} W_{i}^{W}\left(\Omega_{k}\right)}{2 N_{i}\left(\Omega_{k}\right)}\right) \\
& -\zeta_{7, i+1}^{k} u_{i} \\
& \Delta \zeta_{2, i}^{k}=-A_{1}-\zeta_{2, i+1}^{k}\left(1-\sum_{j=1}^{p} \frac{\alpha_{j k} V_{i}^{M}\left(\Omega_{j}\right)+\beta_{j k} D_{i}^{M}\left(\Omega_{j}\right)+\eta_{j k} W_{i}^{M}\left(\Omega_{j}\right)}{N_{i}\left(\Omega_{j}\right)+N_{i}\left(\Omega_{k}\right)}-d_{k}-u_{i}\right) \\
& +\zeta_{1, i+1}^{k} \frac{\alpha_{k k} V_{i}^{M}\left(\Omega_{k}\right)}{2 N_{i}\left(\Omega_{k}\right)}+\zeta_{3, i+1}^{k} \frac{\beta_{k k} D_{i}^{M}\left(\Omega_{k}\right)}{2 N_{i}\left(\Omega_{k}\right)}+\zeta_{5, i+1}^{k} \frac{\eta_{k k} W_{i}^{M}\left(\Omega_{k}\right)}{2 N_{i}\left(\Omega_{k}\right)} \\
& -\left(\zeta_{7, i+1}^{k}+\zeta_{8, i+1}^{k}\right)\left(\sum_{j=1}^{p} \frac{\alpha_{j k}^{k} V_{i}^{M}\left(\Omega_{j}\right)+\beta_{j k}^{k} D_{i}^{M}\left(\Omega_{j}\right)+\eta_{j k}^{k} W_{i}^{M}\left(\Omega_{j}\right)}{N_{i}\left(\Omega_{j}\right)+N_{i}\left(\Omega_{k}\right)}+\frac{\alpha_{k k}^{k} V_{i}^{M}\left(\Omega_{k}\right)+\beta_{k k}^{k} D_{i}^{M}\left(\Omega_{k}\right)+\eta_{k k}^{k} W_{i}^{M}\left(\Omega_{k}\right)}{2 N_{i}\left(\Omega_{k}\right)}\right) \\
& -\zeta_{8, i+1}^{k} u_{i} \\
& \Delta \zeta_{3, i}^{k}=-\zeta_{3, i+1}^{k}\left(1-\sum_{j=1}^{p} \frac{\beta_{k j} V_{i}^{W}\left(\Omega_{j}\right)+\mu_{k j} D_{i}^{W}\left(\Omega_{j}\right)+\theta_{k j} W_{i}^{W}\left(\Omega_{j}\right)}{N_{i}\left(\Omega_{k}\right)+N_{i}\left(\Omega_{j}\right)}-d_{k}-v_{i}\right) \\
& +\zeta_{2, i+1}^{k} \frac{\beta_{k k} V_{i}^{W}\left(\Omega_{k}\right)}{2 N_{i}\left(\Omega_{k}\right)}+\zeta_{4, i+1}^{k} \frac{\mu_{k k} D_{i}^{W}\left(\Omega_{k}\right)}{2 N_{i}\left(\Omega_{k}\right)}+\zeta_{6, i+1}^{k} \frac{\theta_{k k} W_{i}^{W}\left(\Omega_{k}\right)}{2 N_{i}\left(\Omega_{k}\right)} \\
& -\left(\zeta_{7, i+1}^{k}+\zeta_{8, i+1}^{k}\right)\left(\sum_{j=1}^{p} \frac{\beta_{k j}^{k} V_{i}^{W}\left(\Omega_{j}\right)+\mu_{k j}^{k} D_{i}^{W}\left(\Omega_{j}\right)+\theta_{k j}^{k} W_{i}^{W}\left(\Omega_{j}\right)}{N_{i}\left(\Omega_{k}\right)+N_{i}\left(\Omega_{j}\right)}+\frac{\beta_{k k}^{k} V_{i}^{W}\left(\Omega_{k}\right)+\mu_{k k}^{k} D_{i}^{W}\left(\Omega_{k}\right)+\theta_{k k}^{k} W_{i}^{W}\left(\Omega_{k}\right)}{2 N_{i}\left(\Omega_{k}\right)}\right) \\
& -\zeta_{7, i+1}^{k} v_{i} \\
& \Delta \zeta_{4, i}^{k}=-A_{2}-\zeta_{4, i+1}^{k}\left(1-\sum_{j=1}^{p} \frac{\gamma_{j k} V_{i}^{M}\left(\Omega_{j}\right)+\mu_{j k} D_{i}^{M}\left(\Omega_{j}\right)+v_{j k} W_{i}^{M}\left(\Omega_{j}\right)}{N_{i}\left(\Omega_{j}\right)+N_{i}\left(\Omega_{k}\right)}-d_{k}-v_{i}\right) \\
& +\zeta_{1, i+1}^{k} \frac{\gamma_{k k} V_{i}^{M}\left(\Omega_{k}\right)}{2 N_{i}\left(\Omega_{k}\right)}+\zeta_{3, i+1}^{k} \frac{\mu_{k k} D_{i}^{M}\left(\Omega_{k}\right)}{2 N_{i}\left(\Omega_{k}\right)}+\zeta_{5, i+1}^{k} \frac{\nu_{k k} W_{i}^{M}\left(\Omega_{k}\right)}{2 N_{i}\left(\Omega_{k}\right)} \\
& -\left(\zeta_{7, i+1}^{k}+\zeta_{8, i+1}^{k}\right)\left(\sum_{j=1}^{p} \frac{\gamma_{j k}^{k} V_{i}^{M}\left(\Omega_{j}\right)+\mu_{j k}^{k} D_{i}^{M}\left(\Omega_{j}\right)+\gamma_{j k}^{k} W_{i}^{M}\left(\Omega_{j}\right)}{N_{i}\left(\Omega_{j}\right)+N_{i}\left(\Omega_{k}\right)}+\frac{\gamma_{k k}^{k} V_{i}^{M}\left(\Omega_{k}\right)+\mu_{k k}^{k} D_{i}^{M}\left(\Omega_{k}\right)+v_{k k}^{k} W_{i}^{M}\left(\Omega_{k}\right)}{2 N_{i}\left(\Omega_{k}\right)}\right) \\
& -\zeta_{8, i+1}^{k} v_{i}
\end{aligned}
$$




$$
\begin{aligned}
& \Delta \zeta_{5, i}=-\zeta_{5, i+1}^{k}\left(1-\sum_{j=1}^{p} \frac{\eta_{k j} V_{i}^{W}\left(\Omega_{j}\right)+v_{k j} D_{i}^{W}\left(\Omega_{j}\right)+\sigma_{k j} W_{i}^{W}\left(\Omega_{j}\right)}{N_{i}\left(\Omega_{k}\right)+N_{i}\left(\Omega_{j}\right)}-d_{k}\right) \\
& +\zeta_{2, i+1}^{k} \frac{\eta_{k k} V_{i}^{W}\left(\Omega_{k}\right)}{2 N_{i}\left(\Omega_{k}\right)}+\zeta_{4, i+1}^{k} \frac{v_{k k} D_{i}^{W}\left(\Omega_{k}\right)}{2 N_{i}\left(\Omega_{k}\right)}+\zeta_{6, i+1}^{k} \frac{\sigma_{k k} W_{i}^{W}\left(\Omega_{k}\right)}{2 N_{i}\left(\Omega_{k}\right)} \\
& -\left(\zeta_{7, i+1}^{k}+\zeta_{8, i+1}^{k}\right)\left(\sum_{j=1}^{p} \frac{\eta_{k j}^{k} V_{i}^{W}\left(\Omega_{j}\right)+\nu_{k j}^{k} D_{i}^{W}\left(\Omega_{j}\right)+\sigma_{k j}^{k} W_{i}^{W}\left(\Omega_{j}\right)}{N_{i}\left(\Omega_{k}\right)+N_{i}\left(\Omega_{j}\right)}+\frac{\eta_{k k}^{k} V_{i}^{W}\left(\Omega_{k}\right)+v_{k k}^{k} D_{i}^{W}\left(\Omega_{k}\right)+\sigma_{k k}^{k} W_{i}^{W}\left(\Omega_{k}\right)}{2 N_{i}\left(\Omega_{k}\right)}\right), \\
& \Delta \zeta_{6, i}^{k}=-\zeta_{6, i+1}^{k}\left(1-\sum_{j=1}^{p} \frac{\delta_{j k} V_{i}^{M}\left(\Omega_{j}\right)+\theta_{j k} D_{i}^{M}\left(\Omega_{j}\right)+\sigma_{j k} W_{i}^{M}\left(\Omega_{j}\right)}{N_{i}\left(\Omega_{j}\right)+N_{i}\left(\Omega_{k}\right)}-d_{k}\right) \\
& +\zeta_{1, i+1}^{k} \frac{\delta_{k k} V_{i}^{M}\left(\Omega_{k}\right)}{2 N_{i}\left(\Omega_{k}\right)}+\zeta_{3, i+1}^{k} \frac{\theta_{k k} D_{i}^{M}\left(\Omega_{k}\right)}{2 N_{i}\left(\Omega_{k}\right)}+\zeta_{5, i+1}^{k} \frac{\sigma_{k k} W_{i}^{M}\left(\Omega_{k}\right)}{2 N_{i}\left(\Omega_{k}\right)} \\
& -\left(\zeta_{7, i+1}^{k}+\zeta_{8, i+1}^{k}\right)\left(\sum_{j=1}^{p} \frac{\delta_{j k}^{k} V_{i}^{M}\left(\Omega_{j}\right)+\theta_{j k}^{k} D_{i}^{M}\left(\Omega_{j}\right)+\sigma_{j k}^{k} W_{i}^{M}\left(\Omega_{j}\right)}{N_{i}\left(\Omega_{j}\right)+N_{i}\left(\Omega_{k}\right)}+\frac{\delta_{k k}^{k} V_{i}^{M}\left(\Omega_{k}\right)+\theta_{k k}^{k} D_{i}^{M}\left(\Omega_{k}\right)+\sigma_{k k}^{k} W_{i}^{M}\left(\Omega_{k}\right)}{2 N_{i}\left(\Omega_{k}\right)}\right), \\
& \Delta \zeta_{7, i}^{k}=-\zeta_{3, i+1}^{k} \lambda_{k}-\zeta_{6, i+1}^{k} \rho_{k}^{k}-\zeta_{7, i+1}^{k}\left(1-\lambda_{k}-\rho_{k}\right) \\
& \Delta \zeta_{8, i}^{k}=A_{3}-\zeta_{4, i+1}^{k} \lambda_{k}^{k}-\zeta_{5, i+1}^{k} \omega_{k}-\zeta_{8, i+1}^{k}\left(1-\lambda_{k}-\omega_{k}\right),
\end{aligned}
$$

with transversality conditions

$$
\begin{aligned}
& \zeta_{1, N}=0, \\
& \zeta_{2, N}=A_{1}, \\
& \zeta_{3, N}=0, \\
& \zeta_{4, N}=A_{2}, \\
& \zeta_{5, N}=0, \\
& \zeta_{6, N}=0, \\
& \zeta_{7, N}=0, \\
& \zeta_{8, N}=-A_{3} .
\end{aligned}
$$

Furthermore, the optimal control $\left(u_{i}^{*}, v_{i}^{*}\right)$ is given by

$$
\begin{aligned}
& u_{i}^{*}=\min \left\{\max \left\{\frac{V_{i}^{M}\left(\Omega_{k}\right)\left(\zeta_{1, i+1}-\zeta_{7, i+1}\right)+V_{i}^{W}\left(\Omega_{k}\right)\left(\zeta_{2, i+1}-\zeta_{8, i+1}\right)}{\tau_{1}}, u_{\min }\right\}, u_{\max }\right\} . \\
& v_{i}^{*}=\min \left\{\max \left\{\frac{D_{i}^{M}\left(\Omega_{k}\right)\left(\zeta_{3, i+1}-\zeta_{7, i+1}\right)+D_{i}^{W}\left(\Omega_{k}\right)\left(\zeta_{4, i+1}-\zeta_{8, i+1}\right)}{\tau_{2}}, v_{\min }\right\}, v_{\max }\right\},
\end{aligned}
$$

for $i=0, \ldots, N-1$.

Proof. Using Pontryagin et al.'s maximum principle [28] and setting $V^{M *}\left(\Omega_{k}\right), V^{W *}\left(\Omega_{k}\right), M^{M *}\left(\Omega_{k}\right), M^{W *}\left(\Omega_{k}\right)$,
$D^{M *}\left(\Omega_{k}\right), D^{W *}\left(\Omega_{k}\right), W^{M *}\left(\Omega_{k}\right), W^{W *}\left(\Omega_{k}\right)$, and $\left(u^{*}, v^{*}\right)$, we obtain the following adjoint equations: 


$$
\begin{aligned}
& \Delta \zeta_{1, i}^{k}=-\frac{\partial H}{\partial V_{i}^{M}\left(\Omega_{k}\right)} \\
& {\left[\quad \left[\zeta_{1, i+1}\left(1-\sum_{j=1}^{p} \frac{\alpha_{k j} V_{i}^{W}\left(\Omega_{j}\right)+\gamma_{k j} D_{i}^{W}\left(\Omega_{j}\right)+\delta_{k j} W_{i}^{W}\left(\Omega_{j}\right)}{N_{i}\left(\Omega_{k}\right)+N_{i}\left(\Omega_{j}\right)}-d_{k}-u_{i}\right)\right.\right.} \\
& -\zeta_{2, i+1}^{k} \frac{\alpha_{k k}}{2 N_{i}\left(\Omega_{k}\right)} V_{i}^{W}\left(\Omega_{k}\right)-\zeta_{4, i+1}^{k} \frac{\gamma_{k k}}{2 N_{i}\left(\Omega_{k}\right)} D_{i}^{W}\left(\Omega_{k}\right)-\zeta_{6, i+1}^{k} \frac{\delta_{k k}}{2 N_{i}\left(\Omega_{k}\right)} W_{i}^{W}\left(\Omega_{k}\right) \\
& =-\left[+\zeta_{7, i+1}^{k}\left(\sum_{j=1}^{p} \frac{\alpha_{k j}^{k} V_{i}^{W}\left(\Omega_{j}\right)+\gamma_{k j}^{k} D_{i}^{W}\left(\Omega_{j}\right)+\delta_{k j}^{k} W_{i}^{W}\left(\Omega_{j}\right)}{N_{i}\left(\Omega_{k}\right)+N_{i}\left(\Omega_{j}\right)}+\frac{\alpha_{k k}^{k}}{2 N_{i}\left(\Omega_{k}\right)} V_{i}^{W}\left(\Omega_{k}\right)+\frac{\gamma_{k k}^{k}}{2 N_{i}\left(\Omega_{k}\right)} D_{i}^{W}\left(\Omega_{k}\right)+\frac{\delta_{k k}^{k}}{2 N_{i}\left(\Omega_{k}\right)} W_{i}^{W}\left(\Omega_{k}\right)+u_{i}\right)\right. \\
& {\left[+\zeta_{8, i+1}^{k}\left(\sum_{j=1}^{p} \frac{\alpha_{k j}^{k} V_{i}^{W}\left(\Omega_{j}\right)+\gamma_{k j}^{k} D_{i}^{W}\left(\Omega_{j}\right)+\delta_{k j}^{k} W_{i}^{W}\left(\Omega_{j}\right)}{N_{i}\left(\Omega_{k}\right)+N_{i}\left(\Omega_{j}\right)}+\frac{\alpha_{k k}^{k}}{2 N_{i}\left(\Omega_{k}\right)} V_{i}^{W}\left(\Omega_{k}\right)+\frac{\gamma_{k k}^{k}}{2 N_{i}\left(\Omega_{k}\right)} D_{i}^{W}\left(\Omega_{k}\right)+\frac{\delta_{k k}^{k}}{2 N_{i}\left(\Omega_{k}\right)} W_{i}^{W}\left(\Omega_{k}\right)\right)\right]} \\
& =-\zeta_{1, i+1}^{k}\left(1-\sum_{j=1}^{p} \frac{\alpha_{k j} V_{i}^{W}\left(\Omega_{j}\right)+\gamma_{k j} D_{i}^{W}\left(\Omega_{j}\right)+\delta_{k j} W_{i}^{W}\left(\Omega_{j}\right)}{N_{i}\left(\Omega_{k}\right)+N_{i}\left(\Omega_{j}\right)}-d_{k}-u_{i}\right) \\
& +\zeta_{2, i+1}^{k} \frac{\alpha_{k k} V_{i}^{W}\left(\Omega_{k}\right)}{2 N_{i}\left(\Omega_{k}\right)}+\zeta_{4, i+1}^{k} \frac{\gamma_{k k} D_{i}^{W}\left(\Omega_{k}\right)}{2 N_{i}\left(\Omega_{k}\right)}+\zeta_{6, i+1}^{k} \frac{\delta_{k k} W_{i}^{W}\left(\Omega_{k}\right)}{2 N_{i}\left(\Omega_{k}\right)} \\
& -\left(\zeta_{7, i+1}^{k}+\zeta_{8, i+1}^{k}\right)\left(\sum_{j=1}^{p} \frac{\alpha_{k j}^{k} V_{i}^{W}\left(\Omega_{j}\right)+\gamma_{k j}^{k} D_{i}^{W}\left(\Omega_{j}\right)+\delta_{k j}^{k} W_{i}^{W}\left(\Omega_{j}\right)}{N_{i}\left(\Omega_{k}\right)+N_{i}\left(\Omega_{j}\right)}+\frac{\alpha_{k k}^{k} V_{i}^{W}\left(\Omega_{k}\right)+\gamma_{k k}^{k} D_{i}^{W}\left(\Omega_{k}\right)+\delta_{k k}^{k} W_{i}^{W}\left(\Omega_{k}\right)}{2 N_{i}\left(\Omega_{k}\right)}\right)-\zeta_{7, i+1}^{k} u_{i},
\end{aligned}
$$

$$
\begin{aligned}
\Delta \zeta_{2, i}^{k}= & -\frac{\partial H}{\partial V_{i}^{W}\left(\Omega_{k}\right)} \\
= & -\left[\begin{array}{c}
A_{1}-\zeta_{1, i+1}^{k} \frac{\alpha_{k k}}{2 N_{i}\left(\Omega_{k}\right)} V_{i}^{M}\left(\Omega_{k}\right) \zeta_{2, i+1}\left(1-\sum_{j=1}^{p} \frac{\alpha_{j k} V_{i}^{M}\left(\Omega_{j}\right)+\beta_{j k} D_{i}^{M}\left(\Omega_{j}\right)+\eta_{j k} W_{i}^{M}\left(\Omega_{j}\right)}{N_{i}\left(\Omega_{k}\right)+N_{i}\left(\Omega_{j}\right)}-d_{k}-u_{i}\right) \\
-\zeta_{3, i+1}^{k} \frac{\beta_{k k}}{2 N_{i}\left(\Omega_{k}\right)} D_{i}^{M}\left(\Omega_{k}\right)-\zeta_{5, i+1}^{k} \frac{\eta_{k k}}{2 N_{i}\left(\Omega_{k}\right)} W_{i}^{M}\left(\Omega_{k}\right) \\
+\zeta_{7, i+1}^{k}\left(\frac{\alpha_{k k}^{k}}{2 N_{i}\left(\Omega_{k}\right)} V_{i}^{M}\left(\Omega_{k}\right)+\sum_{j=1}^{p} \frac{\alpha_{j k}^{k} V_{i}^{M}\left(\Omega_{j}\right)+\beta_{j k}^{k} D_{i}^{M}\left(\Omega_{j}\right)+\eta_{j k}^{k} W_{i}^{M}\left(\Omega_{j}\right)}{N_{i}\left(\Omega_{k}\right)+N_{i}\left(\Omega_{j}\right)}+\frac{\beta_{k k}^{k}}{2 N_{i}\left(\Omega_{k}\right)} D_{i}^{M}\left(\Omega_{k}\right)+\frac{\eta_{k k}^{k}}{2 N_{i}\left(\Omega_{k}\right)} W_{i}^{M}\left(\Omega_{k}\right)\right) \\
+\zeta_{8, i+1}^{k}\left(\frac{\alpha_{k k}^{k}}{2 N_{i}\left(\Omega_{k}\right)} V_{i}^{M}\left(\Omega_{k}\right)+\sum_{j=1}^{p} \frac{\alpha_{j k}^{k} V_{i}^{M}\left(\Omega_{j}\right)+\beta_{j k}^{k} D_{i}^{M}\left(\Omega_{j}\right)+\eta_{j k}^{k} W_{i}^{M}\left(\Omega_{j}\right)}{N_{i}\left(\Omega_{k}\right)+N_{i}\left(\Omega_{j}\right)}+\frac{\beta_{k k}^{k}}{2 N_{i}\left(\Omega_{k}\right)} D_{i}^{M}\left(\Omega_{k}\right)+\frac{\eta_{k k}^{k}}{2 N_{i}\left(\Omega_{k}\right)} W_{i}^{M}\left(\Omega_{k}\right)+u_{i}\right)
\end{array}\right] \\
= & -A_{1}-\zeta_{2, i+1}^{k}\left(1-\sum_{j=1}^{p} \frac{\alpha_{j k} V_{i}^{M}\left(\Omega_{j}\right)+\beta_{j k} D_{i}^{M}\left(\Omega_{j}\right)+\eta_{j k} W_{i}^{M}\left(\Omega_{j}\right)}{N_{i}\left(\Omega_{j}\right)+N_{i}\left(\Omega_{k}\right)}-d_{k}-u_{i}\right) \\
& +\zeta_{1, i+1}^{k} \frac{\alpha_{k k} V_{i}^{M}\left(\Omega_{k}\right)}{2 N_{i}\left(\Omega_{k}\right)}+\zeta_{3, i+1}^{k} \frac{\beta_{k k} D_{i}^{M}\left(\Omega_{k}\right)}{2 N_{i}\left(\Omega_{k}\right)}+\zeta_{5, i+1}^{k} \frac{\eta_{k k} W_{i}^{M}\left(\Omega_{k}\right)}{2 N_{i}\left(\Omega_{k}\right)} \\
& -\left(\zeta_{7, i+1}^{k}+\zeta_{8, i+1}^{k}\right)\left(\sum_{j=1}^{p} \frac{\alpha_{j k}^{k} V_{i}^{M}\left(\Omega_{j}\right)+\beta_{j k}^{k} D_{i}^{M}\left(\Omega_{j}\right)+\eta_{j k}^{k} W_{i}^{M}\left(\Omega_{j}\right)}{N_{i}\left(\Omega_{j}\right)+N_{i}\left(\Omega_{k}\right)}+\frac{\alpha_{k k}^{k} V_{i}^{M}\left(\Omega_{k}\right)+\beta_{k k}^{k} D_{i}^{M}\left(\Omega_{k}\right)+\eta_{k k}^{k} W_{i}^{M}\left(\Omega_{k}\right)}{2 N_{i}\left(\Omega_{k}\right)}\right. \\
& -\zeta_{8, i+1}^{k} u_{i},
\end{aligned}
$$




$$
\begin{aligned}
& \Delta \zeta_{3, i}^{k}=-\frac{\partial H}{\partial D_{i}^{M}\left(\Omega_{k}\right)} \\
& {\left[-\zeta_{2, i+1}^{k} \frac{\beta_{k k}}{2 N_{i}\left(\Omega_{k}\right)} V_{i}^{W}\left(\Omega_{k}\right)+\zeta_{3, i+1}\left(1-\sum_{j=1}^{p} \frac{\beta_{k j} V_{i}^{W}\left(\Omega_{j}\right)+\mu_{j k} D_{i}^{W}\left(\Omega_{j}\right)+\theta_{k j} W_{i}^{W}\left(\Omega_{j}\right)}{N_{i}\left(\Omega_{k}\right)+N_{i}\left(\Omega_{j}\right)}-d_{k}-v_{i}\right)\right.} \\
& -\zeta_{4, i+1}^{k} \frac{\mu_{k k}}{2 N_{i}\left(\Omega_{k}\right)} D_{i}^{W}\left(\Omega_{k}\right)-\zeta_{6, i+1}^{k} \frac{\theta_{k k}}{2 N_{i}\left(\Omega_{k}\right)} W_{i}^{W}\left(\Omega_{k}\right) \\
& =-+\zeta_{7, i+1}^{k}\left(\frac{\beta_{k k}^{k}}{2 N_{i}\left(\Omega_{k}\right)} V_{i}^{W}\left(\Omega_{k}\right)+\sum_{j=1}^{p} \frac{\beta_{k j}^{k} V_{i}^{W}\left(\Omega_{j}\right)+\mu_{k j}^{k} D_{i}^{W}\left(\Omega_{j}\right)+\theta_{k j}^{k} W_{i}^{W}\left(\Omega_{j}\right)}{N_{i}\left(\Omega_{k}\right)+N_{i}\left(\Omega_{j}\right)}+\frac{\mu_{k k}^{k}}{2 N_{i}\left(\Omega_{k}\right)} D_{i}^{W}\left(\Omega_{k}\right)+\frac{\theta_{k k}^{k}}{2 N_{i}\left(\Omega_{k}\right)} W_{i}^{W}\left(\Omega_{k}\right)+v_{i}\right) \\
& \left.+\zeta_{8, i+1}^{k}\left(\frac{\beta_{k k}^{k}}{2 N_{i}\left(\Omega_{k}\right)} V_{i}^{W}\left(\Omega_{k}\right)+\sum_{j=1}^{p} \frac{\beta_{k j}^{k} V_{i}^{W}\left(\Omega_{j}\right)+\mu_{k j}^{k} D_{i}^{W}\left(\Omega_{j}\right)+\theta_{k j}^{k} W_{i}^{W}\left(\Omega_{j}\right)}{N_{i}\left(\Omega_{k}\right)+N_{i}\left(\Omega_{j}\right)}+\frac{\mu_{k k}^{k}}{2 N_{i}\left(\Omega_{k}\right)} D_{i}^{W}\left(\Omega_{k}\right)+\frac{\theta_{k k}^{k}}{2 N_{i}\left(\Omega_{k}\right)} W_{i}^{W}\left(\Omega_{k}\right)\right)\right] \\
& =-\zeta_{3, i+1}^{k}\left(1-\sum_{j=1}^{p} \frac{\beta_{k j} V_{i}^{W}\left(\Omega_{j}\right)+\mu_{k j} D_{i}^{W}\left(\Omega_{j}\right)+\theta_{k j} W_{i}^{W}\left(\Omega_{j}\right)}{N_{i}\left(\Omega_{k}\right)+N_{i}\left(\Omega_{j}\right)}-d_{k}-v_{i}\right) \\
& +\zeta_{2, i+1}^{k} \frac{\beta_{k k} V_{i}^{W}\left(\Omega_{k}\right)}{2 N_{i}\left(\Omega_{k}\right)}+\zeta_{4, i+1}^{k} \frac{\mu_{k k} D_{i}^{W}\left(\Omega_{k}\right)}{2 N_{i}\left(\Omega_{k}\right)}+\zeta_{6, i+1}^{k} \frac{\theta_{k k} W_{i}^{W}\left(\Omega_{k}\right)}{2 N_{i}\left(\Omega_{k}\right)} \\
& -\left(\zeta_{7, i+1}^{k}+\zeta_{8, i+1}^{k}\right)\left(\sum_{j=1}^{p} \frac{\beta_{k j}^{k} V_{i}^{W}\left(\Omega_{j}\right)+\mu_{k j}^{k} D_{i}^{W}\left(\Omega_{j}\right)+\theta_{k j}^{k} W_{i}^{W}\left(\Omega_{j}\right)}{N_{i}\left(\Omega_{k}\right)+N_{i}\left(\Omega_{j}\right)}+\frac{\beta_{k k}^{k} V_{i}^{W}\left(\Omega_{k}\right)+\mu_{k k}^{k} D_{i}^{W}\left(\Omega_{k}\right)+\theta_{k k}^{k} W_{i}^{W}\left(\Omega_{k}\right)}{2 N_{i}\left(\Omega_{k}\right)}\right) \\
& -\zeta_{7, i+1}^{k} v_{i}
\end{aligned}
$$

$$
\begin{aligned}
\Delta \zeta_{4, i}^{k}= & -\frac{\partial H}{\partial D_{i}^{W}\left(\Omega_{k}\right)} \\
= & -\left[\begin{array}{c}
A_{2}-\zeta_{1, i+1}^{k} \frac{\gamma_{k k}}{2 N_{i}\left(\Omega_{k}\right)} V_{i}^{M}\left(\Omega_{k}\right)-\zeta_{3, i+1}^{k} \frac{\mu_{k k}}{2 N_{i}\left(\Omega_{k}\right)} D_{i}^{M}\left(\Omega_{k}\right)+\zeta_{4, i+1}\left(1-\sum_{j=1}^{p} \frac{\gamma_{j k} V_{i}^{M}\left(\Omega_{j}\right)+\mu_{j k} D_{i}^{M}\left(\Omega_{j}\right)+\gamma_{j k} W_{i}^{M}\left(\Omega_{j}\right)}{N_{i}\left(\Omega_{k}\right)+N_{i}\left(\Omega_{j}\right)}-d_{k}-v_{i}\right) \\
-\zeta_{5, i+1}^{k} \frac{\gamma_{k k}}{2 N_{i}\left(\Omega_{k}\right)} W_{i}^{M}\left(\Omega_{k}\right)+\zeta_{7, i+1}^{k}\left(\frac{\gamma_{k k}}{2 N_{i}\left(\Omega_{k}\right)} V_{i}^{M}\left(\Omega_{k}\right)+\frac{\mu_{k k}^{k}}{2 N_{i}\left(\Omega_{k}\right)} D_{i}^{M}\left(\Omega_{k}\right)+\sum_{j=1}^{p} \frac{\gamma_{j k}^{k} V_{i}^{M}\left(\Omega_{j}\right)+\mu_{j k}^{k} D_{i}^{M}\left(\Omega_{j}\right)+\gamma_{j k}^{k} W_{i}^{M}\left(\Omega_{j}\right)}{N_{i}\left(\Omega_{k}\right)+N_{i}\left(\Omega_{j}\right)}+\frac{v_{k k}^{k}}{2 N_{i}\left(\Omega_{k}\right)} W_{i}^{M}\left(\Omega_{k}\right)\right) \\
+\zeta_{8, i+1}^{k}\left(\frac{\gamma_{k k}}{2 N_{i}\left(\Omega_{k}\right)} V_{i}^{M}\left(\Omega_{k}\right)+\frac{\mu_{k k}^{k}}{2 N_{i}\left(\Omega_{k}\right)} D_{i}^{M}\left(\Omega_{k}\right)+\sum_{j=1}^{p} \frac{\gamma_{j k}^{k} V_{i}^{M}\left(\Omega_{j}\right)+\mu_{j k}^{k} D_{i}^{M}\left(\Omega_{j}\right)+\gamma_{j k}^{k} W_{i}^{M}\left(\Omega_{j}\right)}{N_{i}\left(\Omega_{k}\right)+N_{i}\left(\Omega_{j}\right)}+\frac{v_{k k}^{k}}{2 N_{i}\left(\Omega_{k}\right)} W_{i}^{M}\left(\Omega_{k}\right)+v_{i}\right)
\end{array}\right] \\
= & -A_{2}-\zeta_{4, i+1}^{k}\left(1-\sum_{j=1}^{p} \frac{\gamma_{j k} V_{i}^{M}\left(\Omega_{j}\right)+\mu_{j k} D_{i}^{M}\left(\Omega_{j}\right)+v_{j k} W_{i}^{M}\left(\Omega_{j}\right)}{N_{i}\left(\Omega_{j}\right)+N_{i}\left(\Omega_{k}\right)}-d_{k}-v_{i}\right) \\
& +\zeta_{1, i+1}^{k} \frac{\gamma_{k k} V_{i}^{M}\left(\Omega_{k}\right)}{2 N_{i}\left(\Omega_{k}\right)}+\zeta_{3, i+1}^{k} \frac{\mu_{k k} D_{i}^{M}\left(\Omega_{k}\right)}{2 N_{i}\left(\Omega_{k}\right)}+\zeta_{5, i+1}^{k} \frac{v_{k k} W_{i}^{M}\left(\Omega_{k}\right)}{2 N_{i}\left(\Omega_{k}\right)} \\
& -\left(\zeta_{7, i+1}^{k}+\zeta_{8, i+1}^{k}\right)\left(\sum_{j=1}^{p} \frac{\gamma_{j k}^{k} V_{i}^{M}\left(\Omega_{j}\right)+\mu_{j k}^{k} D_{i}^{M}\left(\Omega_{j}\right)+v_{j k}^{k} W_{i}^{M}\left(\Omega_{j}\right)}{N_{i}\left(\Omega_{j}\right)+N_{i}\left(\Omega_{k}\right)}+\frac{\gamma_{k k}^{k} V_{i}^{M}\left(\Omega_{k}\right)+\mu_{k k}^{k} D_{i}^{M}\left(\Omega_{k}\right)+v_{k k}^{k} W_{i}^{M}\left(\Omega_{k}\right)}{2 N_{i}\left(\Omega_{k}\right)}\right) \\
& -\zeta_{8, i+1}^{k} v_{i},
\end{aligned}
$$




$$
\begin{aligned}
& \Delta \zeta_{5, i}^{k}=-\frac{\partial H}{\partial W_{i}^{M}\left(\Omega_{k}\right)}
\end{aligned}
$$

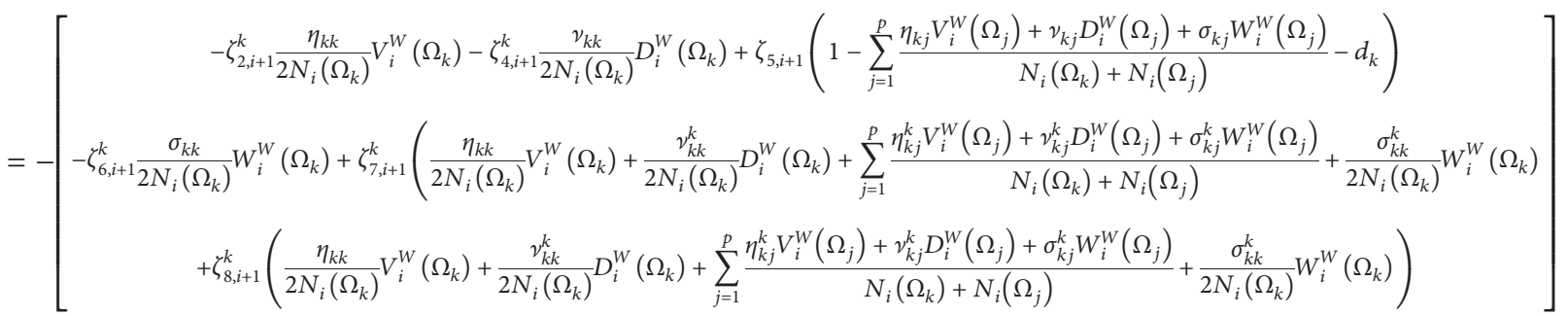

$$
\begin{aligned}
& =-\zeta_{5, i+1}^{k}\left(1-\sum_{j=1}^{p} \frac{\eta_{k j} V_{i}^{W}\left(\Omega_{j}\right)+v_{k j} D_{i}^{W}\left(\Omega_{j}\right)+\sigma_{k j} W_{i}^{W}\left(\Omega_{j}\right)}{N_{i}\left(\Omega_{k}\right)+N_{i}\left(\Omega_{j}\right)}-d_{k}\right) \\
& +\zeta_{2, i+1}^{k} \frac{\eta_{k k} V_{i}^{W}\left(\Omega_{k}\right)}{2 N_{i}\left(\Omega_{k}\right)}+\zeta_{4, i+1}^{k} \frac{\nu_{k k} D_{i}^{W}\left(\Omega_{k}\right)}{2 N_{i}\left(\Omega_{k}\right)}+\zeta_{6, i+1}^{k} \frac{\sigma_{k k} W_{i}^{W}\left(\Omega_{k}\right)}{2 N_{i}\left(\Omega_{k}\right)} \\
& -\left(\zeta_{7, i+1}^{k}+\zeta_{8, i+1}^{k}\right)\left(\sum_{j=1}^{p} \frac{\eta_{k j}^{k} V_{i}^{W}\left(\Omega_{j}\right)+v_{k j}^{k} D_{i}^{W}\left(\Omega_{j}\right)+\sigma_{k j}^{k} W_{i}^{W}\left(\Omega_{j}\right)}{N_{i}\left(\Omega_{k}\right)+N_{i}\left(\Omega_{j}\right)}+\frac{\eta_{k k}^{k} V_{i}^{W}\left(\Omega_{k}\right)+v_{k k}^{k} D_{i}^{W}\left(\Omega_{k}\right)+\sigma_{k k}^{k} W_{i}^{W}\left(\Omega_{k}\right)}{2 N_{i}\left(\Omega_{k}\right)}\right) \text {, }
\end{aligned}
$$

$$
\begin{aligned}
& \Delta \zeta_{6, i}^{k}=-\frac{\partial H}{\partial W_{i}^{W}\left(\Omega_{k}\right)} \\
& =-\left[\begin{array}{c}
-\zeta_{1, i+1}^{k} \frac{\delta_{k k}}{2 N_{i}\left(\Omega_{k}\right)} V_{i}^{M}\left(\Omega_{k}\right)-\zeta_{3, i+1}^{k} \frac{\theta_{k k}}{2 N_{i}\left(\Omega_{k}\right)} D_{i}^{M}\left(\Omega_{k}\right)-\zeta_{5, i+1}^{k} \frac{\sigma_{k k}}{2 N_{i}\left(\Omega_{k}\right)} W_{i}^{M}\left(\Omega_{k}\right)+\zeta_{6, i+1}\left(1-\sum_{j=1}^{p} \frac{\delta_{j k} V_{i}^{M}\left(\Omega_{j}\right)+\theta_{j k} D_{i}^{M}\left(\Omega_{j}\right)+\sigma_{j k} W_{i}^{M}\left(\Omega_{j}\right)}{N_{i}\left(\Omega_{k}\right)+N_{i}\left(\Omega_{j}\right)}-d_{k}\right) \\
+\zeta_{7, i+1}^{k}\left(\frac{\delta_{k k}}{2 N_{i}\left(\Omega_{k}\right)} V_{i}^{M}\left(\Omega_{k}\right)+\frac{\theta_{k k}^{k}}{2 N_{i}\left(\Omega_{k}\right)} D_{i}^{M}\left(\Omega_{k}\right)+\frac{\sigma_{k k}^{k}}{2 N_{i}\left(\Omega_{k}\right)} W_{i}^{M}\left(\Omega_{k}\right)+\sum_{j=1}^{p} \frac{\delta_{j k}^{k} V_{i}^{M}\left(\Omega_{j}\right)+\theta_{j k}^{k} D_{i}^{M}\left(\Omega_{j}\right)+\sigma_{j k}^{k} W_{i}^{M}\left(\Omega_{j}\right)}{N_{i}\left(\Omega_{k}\right)+N_{i}\left(\Omega_{j}\right)}\right) \\
+\zeta_{8, i+1}^{k}\left(\frac{\delta_{k k}}{2 N_{i}\left(\Omega_{k}\right)} V_{i}^{M}\left(\Omega_{k}\right)+\frac{\theta_{k k}^{k}}{2 N_{i}\left(\Omega_{k}\right)} D_{i}^{M}\left(\Omega_{k}\right)\left(+\frac{\sigma_{k k}^{k}}{2 N_{i}\left(\Omega_{k}\right)} W_{i}^{M}\left(\Omega_{k}\right)\right)+\sum_{j=1}^{p} \frac{\delta_{j k}^{k} V_{i}^{M}\left(\Omega_{j}\right)+\theta_{j k}^{k} D_{i}^{M}\left(\Omega_{j}\right)+\sigma_{j k}^{k} W_{i}^{M}\left(\Omega_{j}\right)}{N_{i}\left(\Omega_{k}\right)+N_{i}\left(\Omega_{j}\right)}\right)
\end{array}\right] \\
& =-\zeta_{6, i+1}^{k}\left(1-\sum_{j=1}^{p} \frac{\delta_{j k} V_{i}^{M}\left(\Omega_{j}\right)+\theta_{j k} D_{i}^{M}\left(\Omega_{j}\right)+\sigma_{j k} W_{i}^{M}\left(\Omega_{j}\right)}{N_{i}\left(\Omega_{j}\right)+N_{i}\left(\Omega_{k}\right)}-d_{k}\right) \\
& +\zeta_{1, i+1}^{k} \frac{\delta_{k k} V_{i}^{M}\left(\Omega_{k}\right)}{2 N_{i}\left(\Omega_{k}\right)}+\zeta_{3, i+1}^{k} \frac{\theta_{k k} D_{i}^{M}\left(\Omega_{k}\right)}{2 N_{i}\left(\Omega_{k}\right)}+\zeta_{5, i+1}^{k} \frac{\sigma_{k k} W_{i}^{M}\left(\Omega_{k}\right)}{2 N_{i}\left(\Omega_{k}\right)} \\
& -\left(\zeta_{7, i+1}^{k}+\zeta_{8, i+1}^{k}\right)\left(\sum_{j=1}^{p} \frac{\delta_{j k}^{k} V_{i}^{M}\left(\Omega_{j}\right)+\theta_{j k}^{k} D_{i}^{M}\left(\Omega_{j}\right)+\sigma_{j k}^{k} W_{i}^{M}\left(\Omega_{j}\right)}{N_{i}\left(\Omega_{j}\right)+N_{i}\left(\Omega_{k}\right)}+\frac{\delta_{k k}^{k} V_{i}^{M}\left(\Omega_{k}\right)+\theta_{k k}^{k} D_{i}^{M}\left(\Omega_{k}\right)+\sigma_{k k}^{k} W_{i}^{M}\left(\Omega_{k}\right)}{2 N_{i}\left(\Omega_{k}\right)}\right)
\end{aligned}
$$

$$
\begin{aligned}
& \Delta \zeta_{7, i}^{k}=-\frac{\partial H}{\partial M_{i}^{M}\left(\Omega_{k}\right)}=-\left[\zeta_{3, i+1}^{k} \lambda_{k}+\zeta_{6, i+1}^{k} \rho_{k}^{k}+\zeta_{7, i+1}^{k}\left(1-\left(\lambda_{k}+\rho_{k}\right)\right)\right], \\
& \Delta \zeta_{8, i}^{k}=-\frac{\partial H}{\partial M_{i}^{W}\left(\Omega_{k}\right)}=-\left[-A_{3}+\zeta_{4, i+1}^{k} \lambda_{k}+\zeta_{5, i+1}^{k} \omega_{k}+\zeta_{8, i+1}^{k}\left(1-\left(\lambda_{k}+\omega_{k}\right)\right)\right],
\end{aligned}
$$


with transversality conditions:

$$
\begin{aligned}
& \zeta_{1, N}=0, \\
& \zeta_{2, N}=A_{1}, \\
& \zeta_{3, N}=0, \\
& \zeta_{4, N}=A_{2}, \\
& \zeta_{5, N}=0, \\
& \zeta_{6, N}=0, \\
& \zeta_{7, N}=0 \\
& \zeta_{8, N}=-A_{3} .
\end{aligned}
$$

To obtain the optimality conditions, we take the variation with respect to control $\left(u_{i}^{*}, v_{i}^{*}\right)$ and set it equal to zero:

$$
\begin{aligned}
\frac{\partial H}{\partial u_{i}}= & \tau_{1} u_{i}-\zeta_{1, i+1}^{k} V_{i}^{M}\left(\Omega_{k}\right)-\zeta_{2, i+1}^{k} V_{i}^{W}\left(\Omega_{k}\right)+\zeta_{7, i+1}^{k} V_{i}^{M}\left(\Omega_{k}\right) \\
& +\zeta_{8, i+1}^{k} V_{i}^{W}\left(\Omega_{k}\right)=0, \\
\frac{\partial H}{\partial v_{i}}= & \tau_{2} u_{i}-\zeta_{3, i+1}^{k} D_{i}^{M}\left(\Omega_{k}\right)-\zeta_{4, i+1}^{k} D_{i}^{W}\left(\Omega_{k}\right)+\zeta_{7, i+1}^{k} D_{i}^{M}\left(\Omega_{k}\right) \\
& +\zeta_{8, i+1}^{k} D_{i}^{W}\left(\Omega_{k}\right)=0 .
\end{aligned}
$$

Then, we obtain the optimal control:

$$
\begin{aligned}
& u_{i}^{*}=\frac{V_{i}^{M}\left(\Omega_{k}\right)\left(\zeta_{1, i+1}^{k}-\zeta_{7, i+1}^{k}\right)+V_{i}^{W}\left(\Omega_{k}\right)\left(\zeta_{2, i+1}^{k}-\zeta_{8, i+1}^{k}\right)}{\tau_{1}} ; \\
& v_{i}^{*}=\frac{D_{i}^{M}\left(\Omega_{k}\right)\left(\zeta_{3, i+1}^{k}-\zeta_{7, i+1}^{k}\right)+D_{i}^{W}\left(\Omega_{k}\right)\left(\zeta_{4, i+1}^{k}-\zeta_{8, i+1}^{k}\right)}{\tau_{2}} .
\end{aligned}
$$

By the bounds in $\mathcal{U}_{a d}$, it is easy to obtain $\left(u_{i}^{*}, v_{i}^{*}\right)$ in the following form:

$$
\begin{aligned}
& u_{i}^{*}=\min \left\{\max \left\{\frac{V_{i}^{M}\left(\Omega_{k}\right)\left(\zeta_{1, i+1}^{k}-\zeta_{7, i+1}^{k}\right)+V_{i}^{W}\left(\Omega_{k}\right)\left(\zeta_{2, i+1}^{k}-\zeta_{8, i+1}^{k}\right)}{\tau_{1}}, u_{\min }\right\}, u_{\max }\right\}, \\
& v_{i}^{*}=\min \left\{\max \left\{\frac{D_{i}^{M}\left(\Omega_{k}\right)\left(\zeta_{3, i+1}^{k}-\zeta_{7, i+1}^{k}\right)+D_{i}^{W}\left(\Omega_{k}\right)\left(\zeta_{4, i+1}^{k}-\zeta_{8, i+1}^{k}\right)}{\tau_{2}}, u_{\min }\right\}, u_{\max }\right\},
\end{aligned}
$$

for $i=0, \ldots, N-1$.

\section{Discussion}

In this section, we provide numerical simulations to demonstrate our theoretical results in the case when the studied domain represents the assembly of $p$ regions (cities, towns, etc.). The code is written and compiled in MATLAB using the data cited in Table 2 . The optimality systems are solved using an iterative method where, at instant $i$, the states $V_{i}^{M}, V_{i}^{W}, \ldots, M_{i}^{W}$ with an initial guess are obtained based on a progressive scheme in time, and their adjoint variables $\zeta_{l, i+1}^{k}, l=1,2, \ldots, 8$ are obtained based on a regressive scheme in time because of the transversality conditions. Afterward, we update the optimal control values (30) and (31) using the values of state and costate variables obtained in the previous steps. Finally, we execute the previous steps until a tolerance criterion is reached. In order to show the importance of our work and without loss of generality, we consider here that $p=4$. An area with 4 regions was considered: two regions $\Omega_{1}$ and $\Omega_{4}$ represent two urban areas and the regions $\Omega_{2}$ and $\Omega_{3}$ represent the two rural areas. The
High Commissioner for Planning (HCP, public establishment) [14] gave various population statistics according to the regions (urban and rural). The values defined in Table 2 are inspired by data mentioned on page 2 . Generally, the HCP gives the average sum of marriage, divorce, and widow rates between different population areas $\alpha_{k j}, \beta_{k j}, \ldots, \omega_{k}$. And, precisely, one of the main objects of our work is to estimate as close as possible the values of the parameters $\alpha_{k j}$, $\ldots, \omega_{k}$ defined in Table 2.

4.1. Simulations without Controls. In this section, Figure 1 depicts dynamics of the states $V_{i}^{M}, V_{i}^{W}, \ldots, M_{i}^{W}$ in regions $\Omega_{1}, \Omega_{2}, \Omega_{3}$, and $\Omega_{4}$, respectively, in the case when there is yet no control strategy, and we note that in all these figures presented here, simulations give us an idea about the evolution of marital status in each region whether rural or urban and the impact of flows of populations between regions on the dynamics of the marital status of each one.

The study of the evolution of the dynamics of the marital status was spread out over a period of 20 years. In Figure 1, we note that the evolution of the population in regions $\Omega_{1}$ and $\Omega_{4}$ is almost the same and similarly for regions $\Omega_{2}$ and $\Omega_{3}$. 
TABle 2: Parameters values associated with discrete-time systems (9)-(16) and with optimal control problem (18).

$$
\begin{aligned}
V_{0}^{M}= & 4500, V_{0}^{W}=4600, D_{0}^{M}=400 \\
D_{0}^{W}= & 600, W_{0}^{M}=200, W_{0}^{W}=300 \\
M_{0}^{M}= & 2500, M_{0}^{W}=2000 \\
\Lambda_{11}= & 250, \Lambda_{12}=250, d_{1}=0.03 \\
& \left(\alpha_{1 j}^{1}\right)_{1 \leq j \leq 4}=(0.07,0.05,0.06,0.05) \\
& \left(\alpha_{1 j}^{j}\right)_{1 \leq j \leq 4}=(0.06,0.07,0.06,0.08) \\
& \left(\beta_{1 j}^{1}\right)_{1 \leq j \leq 4}=(0.04,0.02,0.03,0.03) \\
& \left(\beta_{1 j}^{j}\right)_{1 \leq j \leq 4}=(0.03,0.04,0.04,0.05) \\
& \left(\gamma_{1 j}^{1}\right)_{1 \leq j \leq 4}=(0.02,0.02,0.02,0.03) \\
& \left(\gamma_{1 j}^{j}\right)_{1 \leq j \leq 4}=(0.02,0.02,0.01,0.02) \\
& \left(\delta_{1 j}^{1}\right)_{1 \leq j \leq 4}=(0.01,0.02,0.01,0.01) \\
& \left(\delta_{1 j}^{j}\right)_{1 \leq j \leq 4}=(0.01,0.01,0.01,0.01) \\
& \left(\eta_{1 j}^{1}\right)_{1 \leq j \leq 4}=(0.03,0.02,0.02,0.03) \\
\Omega_{1}: & \left(\eta_{1 j}^{j}\right)_{1 \leq j \leq 4}=(0.02,0.03,0.02,0.02) \\
& \left(\mu_{1 j}^{1}\right)_{1 \leq j \leq 4}=(0.02,0.02,0.01,0.02) \\
& \left(\mu_{1 j}^{j}\right)_{1 \leq j \leq 4}=(0.01,0.02,0.01,0.01) \\
& \left(\theta_{1 j}^{1}\right)_{1 \leq j \leq 4}=(0.02,0.02,0.01,0.02) \\
& \left(\theta_{1 j}^{j}\right)_{1 \leq j \leq 4}=(0.01,0.02,0.01,0.01) \\
& \left(v_{1 j}^{1}\right)_{1 \leq j \leq 4}=(0.02,0.01,0.01,0.02) \\
& \left(\nu_{1 j}^{j}\right)_{1 \leq j \leq 4}=(0.01,0.02,0.01,0.01) \\
& \left(\sigma_{11}^{1}\right)_{1 \leq j \leq 4}=(0.01,0.01,0.01,0.01) \\
& \left(\sigma_{1 j}^{j}\right)_{1 \leq j \leq 4}=(0.01,0.01,0.01,0.02) \\
\lambda_{1}=(0.003,0.001,0.001,0.004) & \rho_{1}=(0.01,0.01,0.01,0.01), \omega_{1}=0.03
\end{aligned}
$$

$$
\begin{aligned}
& V_{0}^{M}=850, V_{0}^{W}=860, D_{0}^{M}=90 \\
& D_{0}^{W}=110, W_{0}^{M}=40, W_{0}^{W}=50 \\
& M_{0}^{M}=450, M_{0}^{W}=400 \\
& \Lambda_{31}=70, \Lambda_{32}=75, d_{3}=0.03 \\
& \left(\alpha_{3 j}^{1}\right)_{1 \leq j \leq 4}=(0.05,0.06,0.05,0.05) \\
& \left(\alpha_{3 j}^{j}\right)_{1 \leq j \leq 4}=(0.07,0.06,0.07,0.07) \\
& \left(\beta_{3 j}^{3}\right)_{1 \leq j \leq 4}=(0.03,0.04,0.03,0.04) \\
& \left(\beta_{3 j}^{j}\right)_{1 \leq j \leq 4}=(0.03,0.02,0.04,0.03) \\
& \left(\gamma_{3 j}^{3}\right)_{1 \leq j \leq 4}=(0.02,0.02,0.02,0.01) \\
& \left(\gamma_{3 j}^{j}\right)_{1 \leq j \leq 4}=(0.02,0.01,0.02,0.02) \\
& \left(\delta_{3 j}^{3}\right)_{1 \leq j \leq 4}=(0.01,0.01,0.01,0.02) \\
& \left(\delta_{3 j}^{j}\right)_{1 \leq j \leq 4}=(0.01,0.02,0.01,0.01) \\
& \left(\eta_{3 j}^{3}\right)_{1 \leq j \leq 4}=(0.04,0.03,0.03,0.02) \\
& \Omega_{3}:\left(\eta_{3 j}^{j}\right)_{1 \leq j \leq 4}=(0.01,0.02,0.03,0.03) \\
& \left(\mu_{3 j}^{3}\right)_{1 \leq j \leq 4}=(0.02,0.01,0.03,0.02) \\
& \left(\mu_{3 j}^{j}\right)_{1 \leq j \leq 4}=(0.01,0.02,0.01,0.01) \\
& \left(\theta_{3 j}^{3}\right)_{1 \leq j \leq 4}=(0.01,0.01,0.01,0.01) \\
& \left(\theta_{3 j}^{j}\right)_{1 \leq j \leq 4}=(0.01,0.02,0.03,0.02) \\
& \left(\nu_{3 j}^{3}\right)_{1 \leq j \leq 4}=(0.01,0.01,0.01,0.02) \\
& \left(\nu_{3 j}^{j}\right)_{1 \leq j \leq 4}=(0.01,0.02,0.01,0.01) \\
& \left(\sigma_{3 j}^{3}\right)_{1 \leq j \leq 4}=(0.01,0.01,0.01,0.01) \\
& \left(\sigma_{3 j}^{j}\right)_{1 \leq j \leq 4}=(0.01,0.01,0.01,0.02) \\
& \lambda_{3}=(0.01,0.02,0.01,0.01) \\
& \rho_{3}=(0.01,0.01,0.01,0.01), \omega_{3}=0.03
\end{aligned}
$$

$$
\begin{aligned}
& V_{0}^{M}= 1050, V_{0}^{W}=1060, D_{0}^{M}=90 \\
& D_{0}^{W}= 100, W_{0}^{M}=40, W_{0}^{W}=45 \\
& M_{0}^{M}=510, M_{0}^{W}=500 \\
& \Lambda_{21}=60, \Lambda_{22}=65, d_{2}=0.03 \\
& \quad\left(\alpha_{2 j}^{2}\right)_{1 \leq j \leq 4}=(0.06,0.07,0.04,0.06) \\
&\left(\alpha_{2 j}^{j}\right)_{1 \leq j \leq 4}=(0.06,0.06,0.07,0.06) \\
&\left(\beta_{2 j}^{2}\right)_{1 \leq j \leq 4}=(0.03,0.04,0.03,0.03) \\
&\left(\beta_{2 j}^{j}\right)_{1 \leq j \leq 4}=(0.03,0.03,0.04,0.04) \\
&\left(\gamma_{2 j}^{2}\right)_{1 \leq j \leq 4}=(0.02,0.02,0.02,0.02) \\
&\left(\gamma_{2 j}^{j}\right)_{1 \leq j \leq 4}=(0.02,0.01,0.02,0.01) \\
&\left(\delta_{2 j}^{2}\right)_{1 \leq j \leq 4}=(0.01,0.02,0.01,0.01) \\
&\left(\delta_{2 j}^{j}\right)_{1 \leq j \leq 4}=(0.01,0.01,0.02,0.01) \\
&\left(\eta_{2 j}^{2}\right)_{1 \leq j \leq 4}=(0.02,0.02,0.03,0.02) \\
& \Omega_{2}: \quad\left(\eta_{2 j}^{j}\right)_{1 \leq j \leq 4}=(0.03,0.02,0.02,0.04) \\
&\left(\mu_{2 j}^{2}\right)_{1 \leq j \leq 4}=(0.01,0.02,0.02,0.01) \\
&\left(\mu_{2 j}^{j}\right)_{1 \leq j \leq 4}=(0.01,0.02,0.01,0.02) \\
&\left(\theta_{2 j}^{2}\right)_{1 \leq j \leq 4}=(0.01,0.02,0.02,0.03) \\
&\left(\theta_{2 j}^{j}\right)_{1 \leq j \leq 4}=(0.02,0.02,0.01,0.01) \\
&\left(v_{2 j}^{2}\right)_{1 \leq j \leq 4}=(0.01,0.02,0.02,0.01) \\
&\left(v_{2 j}^{j}\right)_{1 \leq j \leq 4}=(0.01,0.01,0.01,0.02) \\
&\left(\sigma_{2 j}^{2}\right)_{1 \leq j \leq 4}=(0.01,0.02,0.01,0.01) \\
&\left(\sigma_{2 j}^{j}\right)_{1 \leq j \leq 4}=(0.01,0.01,0.02,0.01) \\
& \lambda_{2}=(0.002,0.001,0.001,0.004) \\
& \rho_{2}=(0.01,0.01,0.01,0.01), \omega_{2}=0.02
\end{aligned}
$$

$$
\begin{aligned}
V_{0}^{M}= & 5500, V_{0}^{W}=5600, D_{0}^{M}=900 \\
D_{0}^{W}= & 820, W_{0}^{M}=400, W_{0}^{W}=300 \\
M_{0}^{M}= & 3500, M_{0}^{W}=3000 \\
\Lambda_{41}= & 250, \Lambda_{42}=260, d_{3}=0.04 \\
& \left(\alpha_{4 j}^{4}\right)_{1 \leq j \leq 4}=(0.05,0.04,0.06,0.07) \\
& \left(\alpha_{4 j}^{j}\right)_{1 \leq j \leq 4}=(0.07,0.07,0.06,0.05) \\
& \left(\beta_{4 j}^{4}\right)_{1 \leq j \leq 4}=(0.05,0.04,0.04,0.05) \\
& \left(\beta_{4 j}^{j}\right)_{1 \leq j \leq 4}=(0.03,0.02,0.03,0.01) \\
& \left(\gamma_{4 j}^{4}\right)_{1 \leq j \leq 4}=(0.02,0.02,0.02,0.02) \\
& \left(\gamma_{4 j}^{j}\right)_{1 \leq j \leq 4}=(0.02,0.02,0.01,0.01) \\
& \left(\delta_{4 j}^{4}\right)_{1 \leq j \leq 4}=(0.01,0.01,0.02,0.01) \\
& \left(\delta_{4 j}^{j}\right)_{1 \leq j \leq 4}=(0.01,0.02,0.01,0.01) \\
& \left(\eta_{4 j}^{4}\right)_{1 \leq j \leq 4}=(0.03,0.03,0.02,0.04) \\
\Omega_{4}: & \left(\eta_{4 j}^{j}\right)_{1 \leq j \leq 4}=(0.03,0.02,0.03,0.02) \\
& \left(\mu_{4 j}^{4}\right)_{1 \leq j \leq 4}=(0.02,0.01,0.02,0.01) \\
& \left(\mu_{4 j}^{j}\right)_{1 \leq j \leq 4}=(0.01,0.02,0.01,0.01) \\
& \left(\theta_{4 j}^{4}\right)_{1 \leq j \leq 4}=(0.02,0.01,0.02,0.01) \\
& \left(\theta_{4 j}^{j}\right)_{1 \leq j \leq 4}=(0.01,0.02,0.01,0.01) \\
& \left(v_{4 j}^{4}\right)_{1 \leq j \leq 4}=(0.02,0.01,0.02,0.01) \\
& \left(v_{4 j}^{j}\right)_{1 \leq j \leq 4}=(0.01,0.02,0.01,0.01) \\
& \left(\sigma_{4 j}^{4}\right)_{1 \leq j \leq 4}=(0.01,0.01,0.02,0.01) \\
& \left(\sigma_{4 j}^{j}\right)_{1 \leq j \leq 4}=(0.01,0.02,0.01,0.01) \\
\lambda_{4}=(0.001,0.001,0.003,0.003) & \rho_{4}=(0.01,0.01,0.01,0.01), \omega_{4}=0.03
\end{aligned}
$$

$\tau_{1}=2.5 \times 10^{6}, \tau_{2}=3 \times 10^{5}$ 


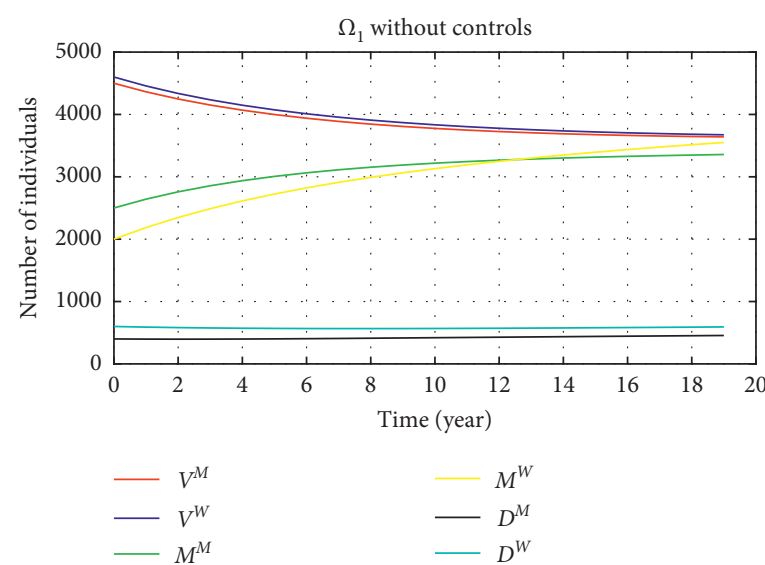

(a)

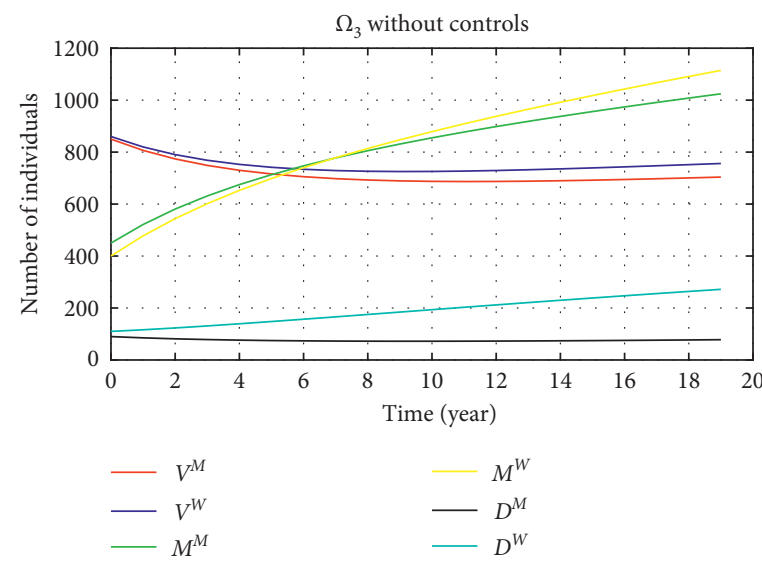

(c)

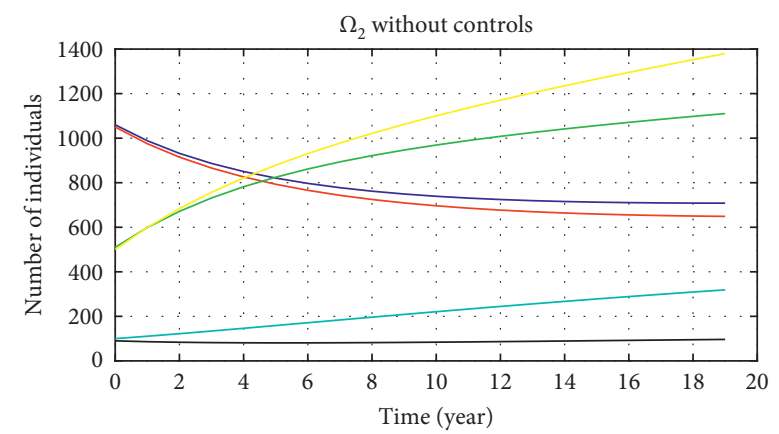

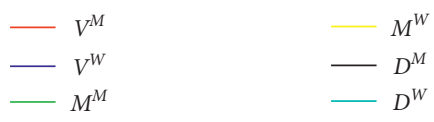

(b)

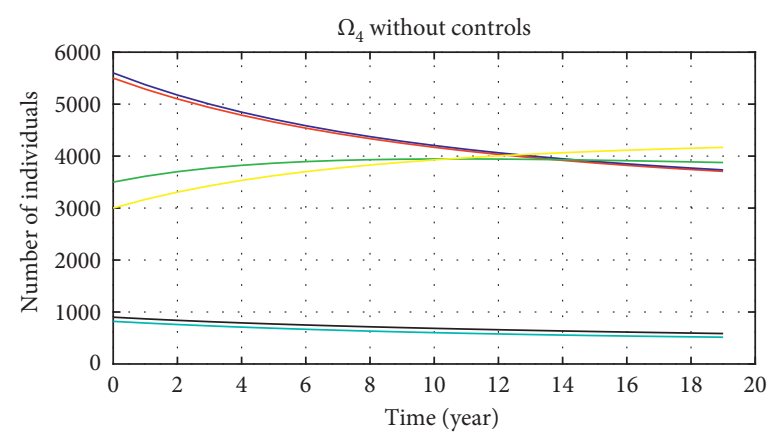

(d)

Figure 1: Time evolution of marital status of family dynamics VMDW without controls.
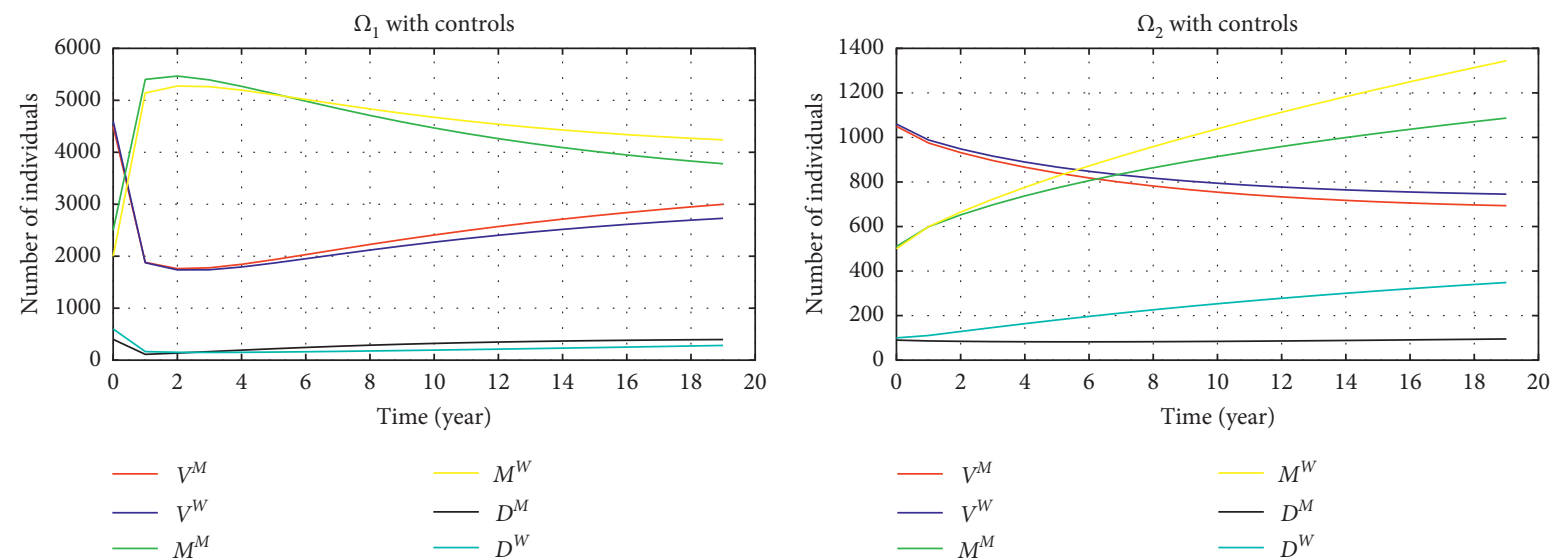

$-V^{M}$
$-V^{W}$
$-M^{M}$

(a)

Figure 2: Continued. 


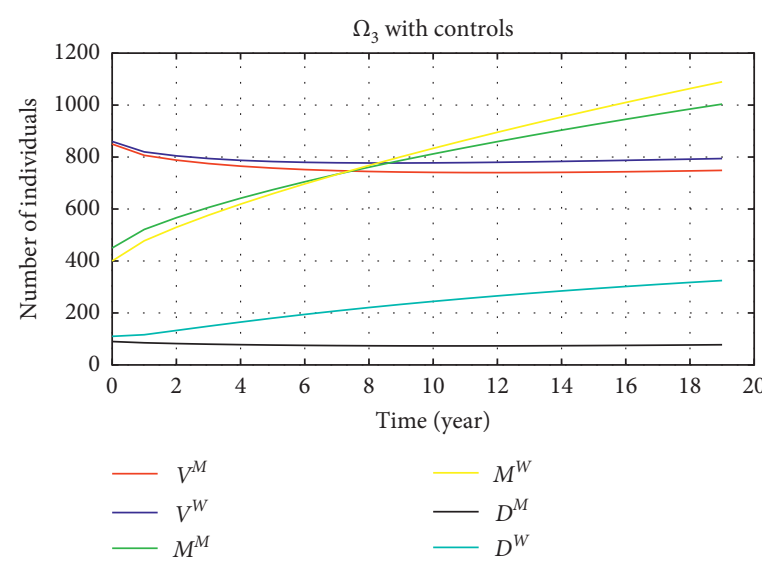

(c)

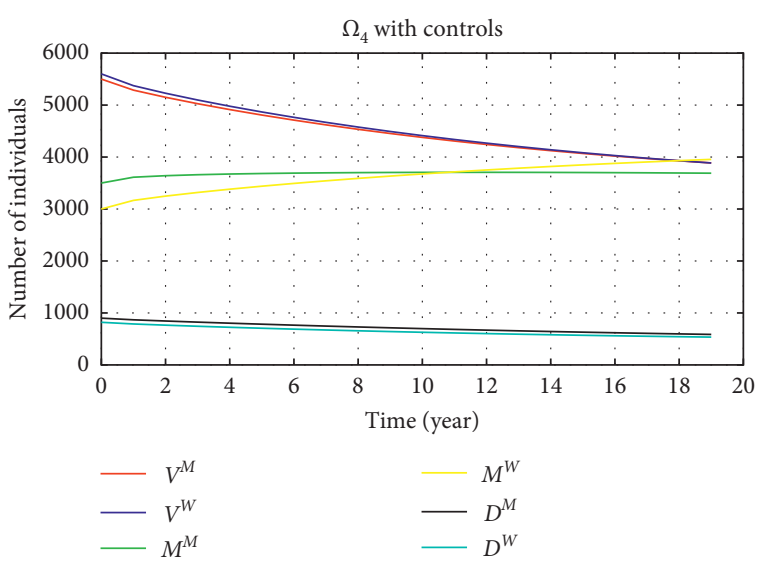

(d)

FIgURE 2: Time evolution of marital status of family dynamics VMDW with controls.

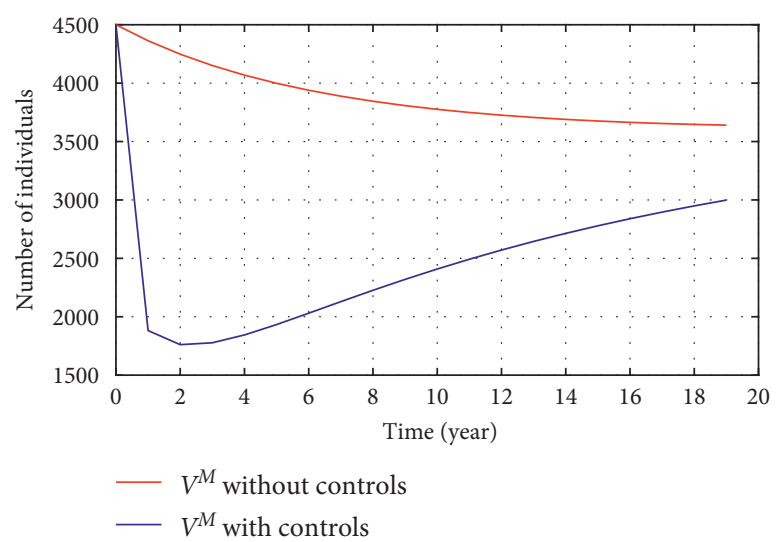

(a)

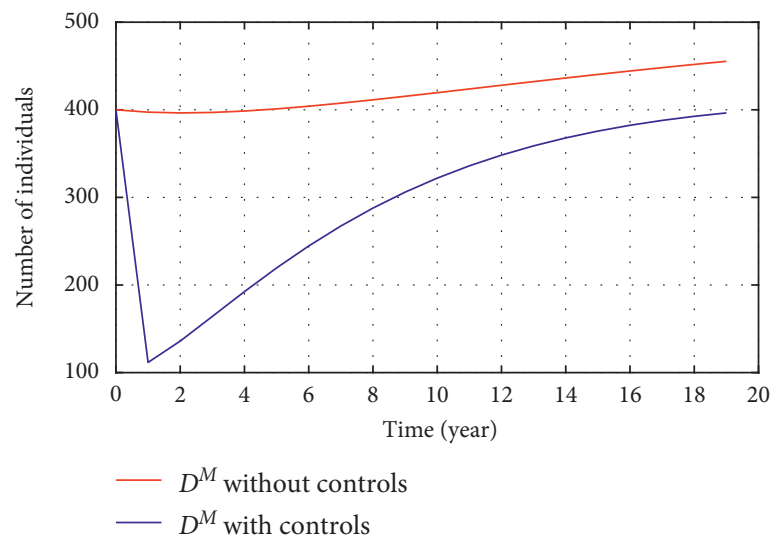

(c)

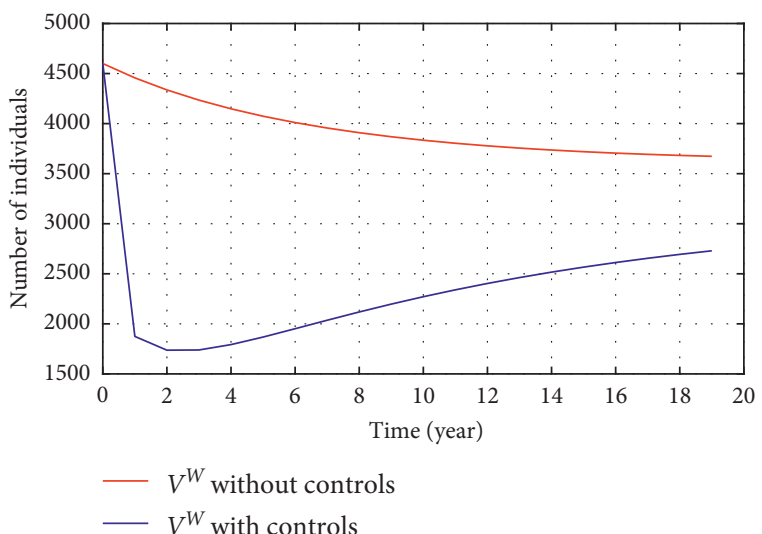

(b)

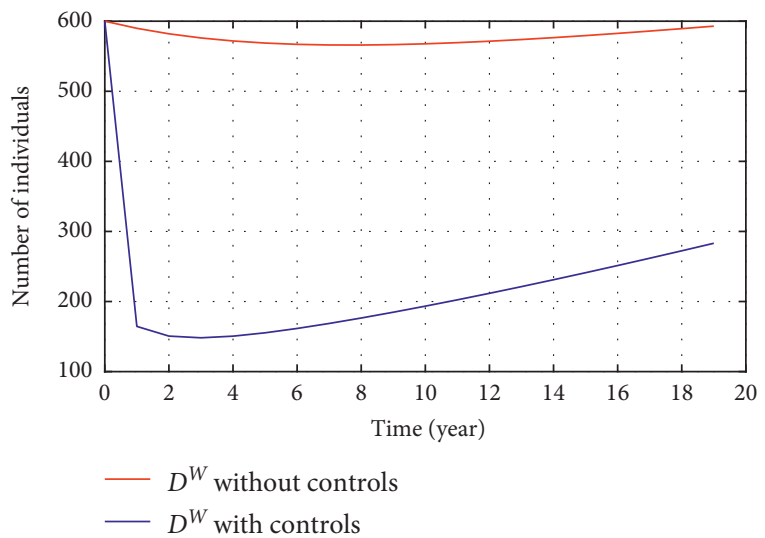

(d)

Figure 3: Time evolution of virgin and divorced populations with and without controls.

In the urban area $\Omega_{1}$, the number of single men is 4500 at time $i=0$, and the number of married men is about 2000 and 400 cases of divorce for men and the same numbers for women, with a birth rate of 250 per year and $0.03 \%$ mortality. The number of virgins declined a little and stabilized after 10 years to 3750 . By cons, for the number of married men and women, there is a slight evolution that reaches after 12 years the number of 3200 married people and finally, for the number of divorces, it remains almost stable throughout the period and remains around 600 divorces. 


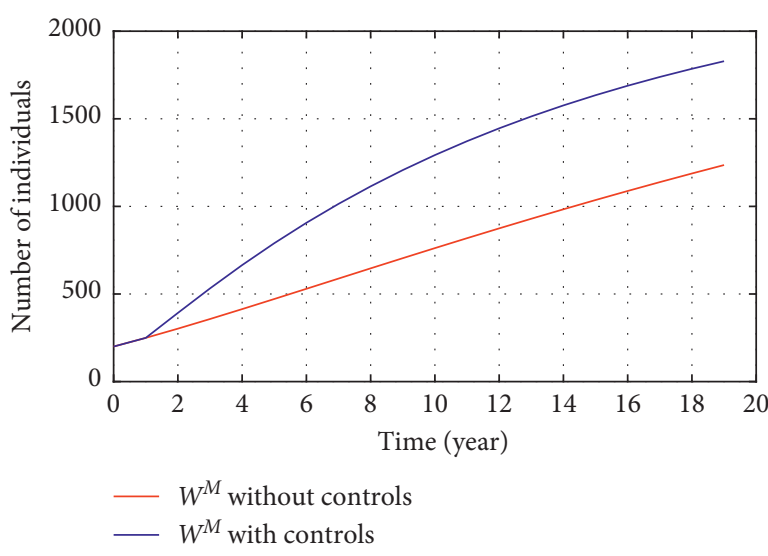

(a)

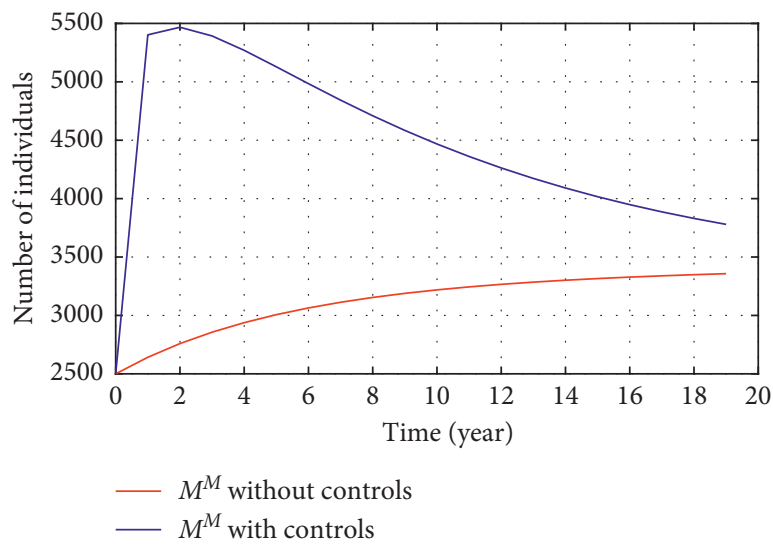

(c)

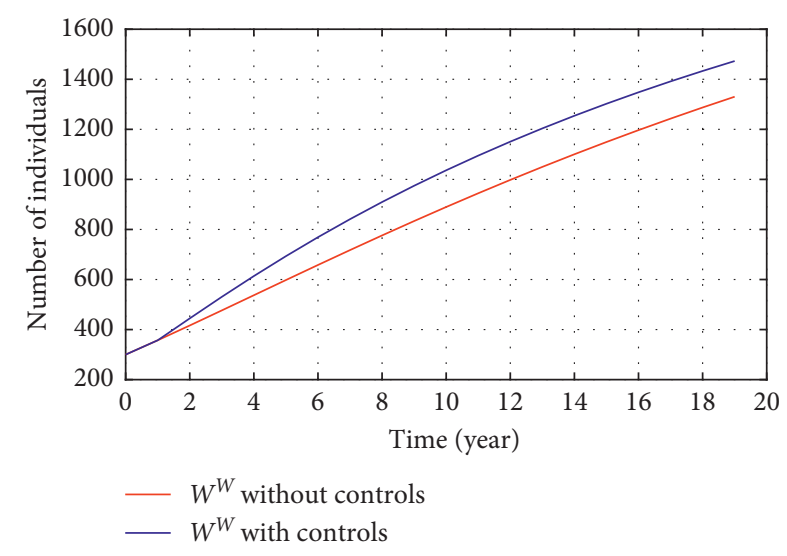

(b)

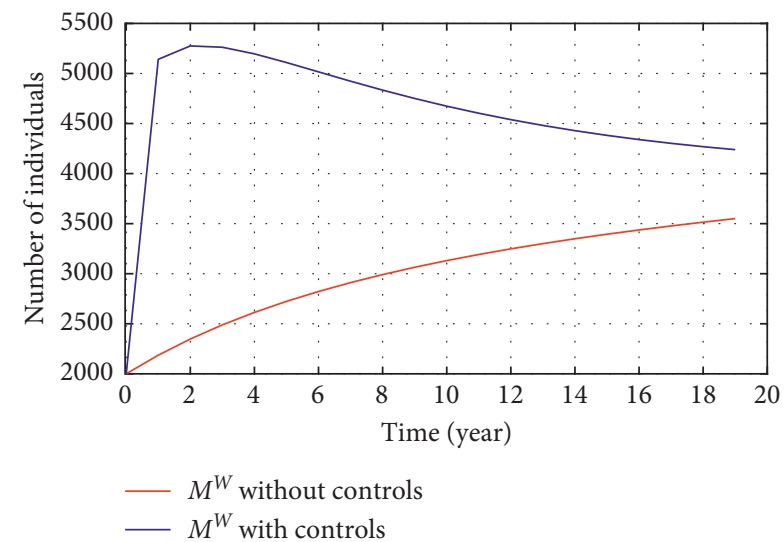

(d)

FIgURE 4: Time evolution of widowed and married populations with and without controls.

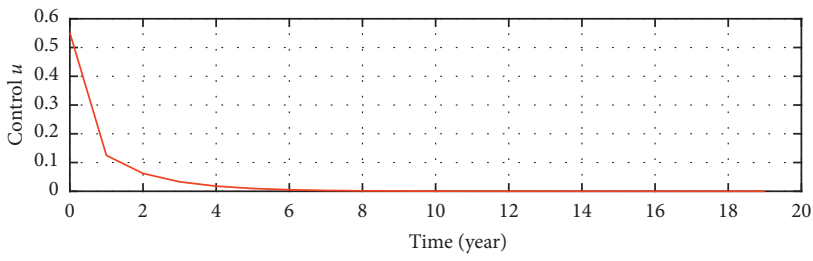

(a)

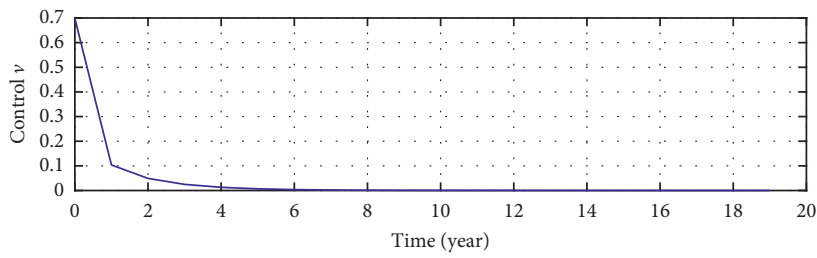

(b)

Figure 5: Time evolution of optimal controls $u^{*}$ and $v^{*}$.

In rural $\Omega_{2}$, the number of unmarried men at time $i=0$ is 1050 , the number of married men is about 500 and 100 divorces for men and the same numbers for women, with a birth rate of 60 per year and $0.03 \%$ mortality. These numbers will be evolved, so the number of virgins has decreased to reach about 700 virgins. The number of married women will experience considerable growth to reach around 1400 for men and 1100 for women. The number of women divorces will witness a slight growth of about 300 divorces and stability for men of almost 100 .

4.2. Simulations with Controls. Considering the critical level of control, we give optimal control sufficient to reduce the number of virgin and divorced individuals and to increase the married individuals in $\Omega_{1}$ and we also studied the effect of controls on other neighboring regions $\Omega_{2}, \Omega_{3}$, and $\Omega_{4}$.

In the following, we can see that the optimal control function has a very desirable effect upon the population of virgin and divorced people which decreases while the married population increases in a consistent way during the length of the process. The time evolution of the respective populations with control is displayed in Figure 2.

Figures 3-5 allow us to compare changes in the number of virgins, divorced, and married individuals before and after the introduction of control.

In a population of virgin men of 4500 individuals (Figure 3(a)) by applying the control law, this number 
decreases rapidly to reach after 3 years the number of 1700 virgins and then goes back up to reach out at the end of the companion awareness 3000 virgin men. This result is similar to virgin women as shown in Figure 3(b).

Figure 3(c) also shows the effect of applying the control law by indicating that the number of divorced men decreases more rapidly at the beginning of treatment to reach 100 male divorces. Then, we notice that the number increases and we can justify it by the fact that the number of divorced people is proportional to the number of married people. Since the number of married individuals increases along the course of treatment, it has been assumed that divorced men remain in $\Omega_{1}$. Unlike divorced women who can return to their region of origin, therefore the number of divorced women that was 600 women decreases rapidly to reach 150 divorced women after 4 years and then increases to 280 divorced women at the end of the companion as shown in Figure 3(d).

Figures 4(c) and 4(d) show that the number of married men and women grows after 2 years and reaches a maximum value of about 2500 for married men and 2000 for married women; then it declines to 3800 for men and 4250 for women at the end of companion. This decline is due to the fact that the number of divorced men and women increases, but it is still quite high as far as the uncontrolled number of married men and women. In Figures 4(a) and 4(b), we show the evolution of widowed men and women; the number increases slightly concerning the number of widowed individuals in a dynamics of the marital system without control strategies.

We also note in Figures 1 and 2 and contrary to the evolution of the dynamics of marital status in $\Omega_{1}$ that the number of virgin and divorced people slightly increases in the regions $\Omega_{2}, \Omega_{3}$, and $\Omega_{4}$ and the number of married people decreases slightly.

Finally, Figure 5 displays the time evolution of the optimal control $\left(u^{*}, v^{*}\right)$.

\section{Conclusions}

In this paper, we consider a discrete-time marital status model; we treat the modelling and control of a system that described the case of a multiregion model. Optimal control is investigated to reduce the number of virgins and the divorced population and to increase the married ones. The first control introduced is supposing the benefits of an awareness campaign to educate virgin men and women about the benefits of marriage for individuals and the society, and the second control characterizes the legal procedures, administrative complications, and the heavy financial and social consequences of divorces. A discrete version of Pontryagin et al.'s maximum principle is done to analyze the optimal control problem and numerical simulation is given to illustrate the obtained results.

\section{Data Availability}

The disciplinary data used to support the findings of this study have been deposited in the Network Repository (http://www.networkrepository.com).

\section{Conflicts of Interest}

The authors declare that they have no conflicts of interest.

\section{References}

[1] R. P. Canale, "An analysis of models describing predator-prey interaction," Biotechnology and Bioengineering, vol. 12, no. 3, pp. 353-378, 1970.

[2] E. D. Conway and J. A. Smoller, "Global analysis of a system of predator-prey equations," SIAM Journal on Applied Mathematics, vol. 46, no. 4, pp. 630-642, 1986.

[3] J. K. Galbraith, The Predator State, Free Press, New York City, NY, USA, 2006.

[4] J. Yan, C. Li, X. Chen, and L. Ren, "Dynamic complexities in 2-dimensional discrete-time predator-prey systems with allee effect in the prey," Discrete Dynamics in Nature and Society, vol. 2016, Article ID 4275372, 14 pages, 2016.

[5] F. B. Agusto, "Optimal isolation control strategies and costeffectiveness analysis of a two-strain avian influenza model," Biosystems, vol. 113, no. 3, pp. 155-164, 2013.

[6] L. J. S. Allen, "Some discrete-time SI, SIR, and SIS epidemic models," Mathematical Biosciences, vol. 124, no. 1, pp. 83-105, 1994.

[7] G. Birkhoff and G. C. Rota, Ordinary Differential Equations, John Wiley \& Sons, Hoboken, NJ, USA, 4th edition, 1989.

[8] F. Brauer and C. Castillo-Chavez, "Mathematical models in population biology and epidemiology," Texts in Applied Mathematics, Vol. 40, Springer, Berlin, Germany, 2001.

[9] L. L. Cavalli-Sforza and M. W. Feldman, "The application of molecular genetic approaches to the study of human evolution," Nature Genetics, vol. 33, no. 3, pp. 266-275, 2003.

[10] U. A. Meyer and U. M. Zanger, "Molecular mechanisms of genetic polymorphisms of drug metabolism," Annual Review of Pharmacology and Toxicology, vol. 37, no. 1, pp. 269-296, 1997.

[11] J. K. Nicholson, "Global systems biology, personalized medicine and molecular epidemiology," Molecular Systems Biology, vol. 2, no. 1, p. 52, 2006.

[12] K. Bennetta and K. Morgan, "Health, social functioning and marital status: stability and change among elderly recently widowed women," International Journal of Geriatric Psychiatry, vol. 7, no. 8, pp. 13-817, 1992.

[13] P. Y. Goodwin, W. D. Mosher, and A. Chandra, "Marriage and cohabitation in the United States: a statistical portrait based on cycle 6 (2002) of the national survey of family growth: national center for health statistics," Vital Health Stat, vol. 23 , no. 28 , pp. 1-45, 2010.

[14] National Documentation Center, High Commission for Planning of Morocco: Marriage and Divorce Moroccan Woman Evolutionary Trends, National Documentation Center, Rabat, Morocco, 2014, http://www.hcp.ma/Mariageet-divorce-de-la-femme-marocaine-Tendances-d-evolution_ a1261.html.

[15] D. Umberson, "Gender, marital status and the social control of health behavior," Social Science \& Medicine, vol. 34, no. 8, pp. 907-917, 1992.

[16] M. L. Usdansky, A. S. London, and J. M. Wilmoth, "Veteran status, race-ethnicity, and marriage among fragile families," Journal of Marriage and Family, vol. 71, no. 3, pp. 768-786, 2009.

[17] I. Waldron, C. C. Weiss, and M. E. Hughes, "Marital status effects on health: are there differences between never married 
women and divorced and separated women?" Social Science \& Medicine, vol. 45, no. 9, pp. 1387-1397, 1997.

[18] J. S. Zax and D. W. Flueck, Marriage, Divorce, Income and Marriage Incentives, University of Colorado, Boulder, CO, USA, 2003.

[19] M. Lhous, M. Rachik, H. Laarabi, and H. Abdelhak, "Discrete mathematical modeling and optimal control of the marital status: the monogamous marriage case," Advances in Difference Equations, vol. 2017, no. 1, 2017.

[20] A. El-Alami Laaroussi, M. Rachik, and M. Elhia, "An optimal control problem for a spatiotemporal SIR model," International Journal of Dynamics and Control, vol. 6, no. 1, 2016.

[21] K. Dabbs, Optimal Control in Discrete Pest Control Models, University of Tennessee, Knoxville, TN, USA, 2010.

[22] K. Hattaf, M. Rachik, S. Saadi, and N. Yousfi, "Optimal control of infection model," Applied Mathematical Sciences, vol. 3, no. 20, pp. 949-958, 2009.

[23] H. R. Joshi, "Optimal control of an HIV immunology model," Optimal Control Applications and Methods, vol. 23, no. 4, pp. 199-213, 2002.

[24] H. Laarabi, M. Rachik, O. El Kahlaoui, and E. Labriji, "Optimal vaccination strategies of an SIR epidemic model with a saturated treatment," Universal Journal of Applied Mathematics, vol. 1, no. 3, pp. 185-191, 2013.

[25] M. Lhous, M. Rachik, and A. Larrache, "Free optimal time control problem for a seir-epidemic model with immigration of infective," International Journal of Computer Applications, vol. 159, no. 3, pp. 1-5, 2017.

[26] M. Lhous, M. Rachik, and A. Larrache, "Optimal vaccination control and free optimal time for a general seir-epidemic model," World Journal of Modelling and Simulation, vol. 15, no. 1, pp. 3-11, 2019.

[27] D. Wandi, R. Hendon, B. Cathey, E. Lancaster, and R. Germick, "Discrete time optimal control applied to pest control problems," Involve, A Journal of Mathematic, vol. 7, no. 4, pp. 479-489, 2014.

[28] L. S. Pontryagin, V. G. Boltyanskii, R. V. Gamkrelidze, and E. F. Mishchenko, The Mathematical Theory of Optimal Processes, Wiley, Hoboken, NJ, USA, 1962.

[29] O. Zakary, M. Rachik, and I. Elmouki, "On the analysis of a multi-regions discrete sir epidemic model: an optimal control approach," International Journal of Dynamics and Control, vol. 5, no. 3, 2017.

[30] D. Jedlicka, Affinographs A Dynamic Method for Assessment of Individuals, Couples, Families, and Households, Springer, Berlin, Germany, 2011.

[31] B. R. Karney and T. N. Bradbury, "Contextual influences on marriage," Current Directions in Psychological Science, vol. 14, no. 4, pp. 171-174, 2005.

[32] D. L. Lukes, Differential Equations: Classical to Controlled, Academic Press, Cambridge, MA, USA, 1982. 